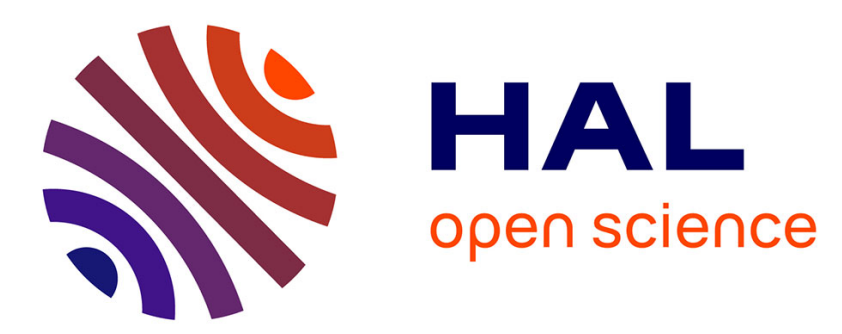

\title{
Anion Transport in Lipid Bilayer Membranes Using Halogen Bonds
}

Stefan Matile, Andreas Vargas Jentzsch

\section{To cite this version:}

Stefan Matile, Andreas Vargas Jentzsch. Anion Transport in Lipid Bilayer Membranes Using Halogen Bonds. Halogen Bonding in Solution, Wiley-VCH Verlag GmbH \& Co. KGaA., 2021, 10.1002/9783527825738.ch6 . hal-03357564

\section{HAL Id: hal-03357564 https://hal.science/hal-03357564}

Submitted on 28 Sep 2021

HAL is a multi-disciplinary open access archive for the deposit and dissemination of scientific research documents, whether they are published or not. The documents may come from teaching and research institutions in France or abroad, or from public or private research centers.
L'archive ouverte pluridisciplinaire HAL, est destinée au dépôt et à la diffusion de documents scientifiques de niveau recherche, publiés ou non, émanant des établissements d'enseignement et de recherche français ou étrangers, des laboratoires publics ou privés. 


\title{
Anion-transport in lipid bilayer membranes using halogen bonds
}

By Stefan Matile and Andreas Vargas Jentzsch*

\section{Affiliation}

Dr. A. Vargas Jentzsch

SAMS Research Group, Institut Charles Sadron, CNRS - UPR 22,

University of Strasbourg, 23 rue du Loess, 67034 Strasbourg (France).

\author{
Prof. S. Matile \\ Department of Organic Chemistry, \\ University of Geneva, Geneva (Switzerland)
}

Keywords: Halogen bonding, Ion transport, Supramolecular chemistry, Organic synthesis, Lipid membrane.

\begin{abstract}
The development of ion transport systems employing halogen bonding for function and structure started less than a decade ago. This line of research was motivated by the intrinsically compatible characteristics of this non-covalent interaction with transmembrane ion transport, leading to a number of macrocyclic and supramolecular approaches to be explored and reported. Amidst more elaborated molecular frameworks, simple perfluoro-1-iodoalkanes have conclusively proven the relevance and suitability of halogen bonding towards this functional application. Indeed, the strength and directionality of the halogen bond, added to a hydrophobic scaffold, was sufficient to enable anion binding and transport across a lipid bilayer membrane. Macrocyclic arrays were advanced to demonstrate multitopic binding and transport, further enlarging the possibilities. Finally, ion channels relying on halogen bonding to enable multiion hoping across the membrane have also been reported. These larger structures could be constructed both using covalent and supramolecular approaches and were the most active transporters exploiting halogen bonding.
\end{abstract}




\section{Introduction}

It is not an exaggeration to claim that the beauty and complexity of natural systems have fascinated scientists for as long as it can remembered. Among numerous examples, the carefully crafted out-of-equilibrium systems that are living organisms hold a prominent place. In this chapter, we will center the discussion on two seemingly opposite phenomena: the existence of highly-isolated compartments, and the necessity to allow transport across this isolation barrier. This barrier is the lipid bilayer membrane that with a typical thickness of about $30-40 \AA$ prevents the exchange of most polar solutes and macromolecules, and is essential to life as we know it. The transport of substrates, notably polar molecules, across the lipid bilayer membrane is assured either in an active or passive manner.

The active transport of any given substrate necessitates the involvement of big protein complexes and typically requires the use of ATP. Several examples exist, with one of the most commonly cited being the sodium-potassium pump (Na+/K+ ATPase) [1]. Such systems, able to facilitate the transport of a given substrate against a concentration gradient, have not yet been mimicked by human-made molecular systems.

Passive transport, in which a given substrate is allowed to diffuse across the bilayer membrane is comparatively simpler and has been the subject of numerous studies since more than 50 years. Depending on their mode of action, many classifications have been proposed. The most common types include ion channels, pores, carriers, and detergents. In contrast to active transport systems, every passive transport mechanism has also been achieved using humanmade molecular systems, with artificial ion channels being the last ones to join the collection about 40 years ago [2,3]. A more detailed discussion of these systems is beyond the scope of this chapter and the reader is referred to the numerous specialized reviews in the field [4-10]. Historically, cation artificial transport systems were developed first either because of the importance of cation transport in biological systems, or due to the early appearance of the remarkable crown-ether motif. Indeed, countless examples of artificial transport systems, many of which can be described as cation channels, have used crown ethers as the main functional design principle [11]. Aside from crown ethers, which are a particular case of Lewis base interactions, other famous macrocycles have also been employed, such as calixarenes [12], and cyclodextrins [13]. Nevertheless, a macrocyclic structure is not required and, for instance, the use of $p$-oligophenyl rigid rods can also permit cation transport by a cation-hopping mechanism using either hydroxy groups [14] or directly taking advantage of the $\pi$-basic nature of the oligophenyl rigid rod [15]. In all cases, the selectivity towards specific cations is dictated by the geometrical and electronic properties of each system. 
Examples of anion artificial transport systems only appeared at a later point in time but have been equally prolific. A vast array of peptidic and non-peptidic families have been reported [3$6,8,16]$. Curiously, while cation transporters are often complex macromolecules, anion transporters can be remarkably simple with regards to their chemical structure, and more often than not follow a carrier mechanism. One possible explanation behind this phenomenon is that anion transport can be enabled by using hydrogen bonding to bind the anion to the molecular carrier.

A few examples are depicted in Scheme 1. For instance, the naturally occurring Prodigiosin 1, its structurally related analogues 2 [17], and related tambjamines 3 [18]. They all take advantage of multiple simultaneous hydrogen bonding interactions to bind chloride and promote transport by a carrier mechanism. Concomitant to their anion transport activities, these compounds - and notably Obatoclax [17] - have shown promising results as anticancer agents. The same design principle can be applied using other equally simple monomers such as squaramides 4 yielding similarly active compounds. Furthermore, anion channels have been reported, which also employ functional hydrogen bonding to achieve both self-assembly and anion transport. Two notable example in this family are isophthalamines 5 and calix[4]arene $\mathbf{6}$ both of which selfassemble in the membrane to promote chloride efflux $[19,20]$.

Nevertheless, the use of hydrogen bonds is not the only possibility to achieve anion transport across a bilayer membrane. A non-exhaustive list of interactions that have been demonstrated to work, in more or less complicated molecular structures, include anion- $\pi$ interactions [21-23], anion-macrodipole interactions [24] and multi-valent ion pairing [25]. Recently, the families of sigma-hole interactions have been introduced [26]. While the discussion in the following will be devoted to examples using halogen bonding, it is worth mentioning here that also chalcogen [27,28] and pnictogen [29] bonds have been very successfully applied to promote anion transport in lipid bilayer membranes.

\subsection{Halogen bonding in the context of ion transport}

This chapter being part of a book on halogen bonding in solution, there is barely any introduction necessary to this slightly less common interaction. Nevertheless, it is important to underline a few considerations that suggested that anion transport using halogen bonding could be a successful endeavor, and promise future applications beyond proof-of-concept examples. First, the vast majority of anion transmembrane transport motifs have employed hydrogen bonds [8]. Yet, the similarities between hydrogen and halogen bonds, like their directionality and strength, are a clear first indication that the later could also be used for anion transport. 
Second, one major difference between these interactions is the hydrophilicity of the typical donor units, with a clear preference of halogen-bond donors - with the notable exception of charge-assisted systems [26,30,31] - to be on the hydrophobic side of the spectra. Both these characteristics, strong binding to anions and hydrophobicity, are considered of great importance when developing anion transport systems [32].

Lastly, a very strong indication of the possibility of using halogen bonding in ion transport systems came from the work of Taylor (Chapter 5) and Beer (Chapter 6): Their work on anion recognition using halogen bonding [33-38] provided strong foundations to the development of the systems described hereafter.

This chapter will be devoted to a comprehensive review of ion transport in lipid bilayer membranes using halogen bonds. It is important to clarify that while most of the discussion will be centered on the description of anion transport, in many cases it also entails the co-transport of the accompanying cation. The main reason for focusing on the anion is, primarily, because in these cases, ion transport is mostly a consequence of binding of the anion using halogen bonding.

\subsection{Organization of this chapter}

The main text of this chapter will describe the specific examples of anion transport that have been reported in the literature. This description will not specifically discuss the techniques employed beyond what is necessary to comprehend the scientific approach and the related findings. As such, we aim to provide the reader with the possibility to skip the highly technical details.

Nevertheless, in most cases, a better understanding of the involved techniques could prove to be useful. To further provide the reader with the possibility to follow the discussion more in detail without having to refer to additional resources, two "Help Boxes" will be introduced where necessary. Such succinct descriptions are not aimed at providing a complete description of the involved techniques, but rather at offering the fundamental elements to understand the experimental and theoretical approaches. References for additional resources will always be provided where convenient.

Regarding the organization of the chapter, we have chosen to forsake a chronological approach and favor organizing the examples according to functional and structural similarities. Accordingly, we will fist discuss examples inspired by traditional macrocyclic approaches, followed by supramolecular assemblies and finishing by reviewing halogen bond channels.

\section{Macrocyclic systems}


Macrocyclic approches have had an historical prevalence in artificial ion transport systems which probably accounts for the fact that the first successful attempts at achieving ion transport employing halogen bonding were based on well-known macrocycles. As it will be described in the following, while such systems have been very useful at demonstrating the feasibility and involvement of halogen bonds in ion transport, the evidence suggests that either further improvements are necessary or that a cyclic array might not be the ideal configuration for halogen-bonding based systems. Two main macrocycles have been reported in this context: calix[4]arenes [39] and oxacalix[2]arene[2]triazine [40].

\subsection{Calix[4]arenes}

Considering the similarities between hydrogen and halogen bonding, it was only natural to use a molecular framework known in the field of anion transport [41,42] and to adapt its molecular structure to enable the formation of halogen bonds, ideally in a cooperative manner. Following this principle, the goal was to use the calixarene scaffold and substitute the lower rim with good halogen-bond donors. From the available options, the use of perfluorophenyl iodide was preferred because it represented the simplest synthetically accessible target.

The calix[4]arene scaffold presented another very useful advantage: the upper rim has been shown to act as a moderately good host towards the tetramethylammonium cation (TMA+) [43], which could be used to tune the affinity of the overall receptor.

\subsubsection{Synthesis}

The bare calix[4]arene (8) was functionalized at the free phenols positions with pentafluorobenzyl bromide, 3-iodobenzyl bromide, 1-(chloromethyl)-2,3,5,6-tetrafluoro-4iodobenzene, 1-(chloromethyl)-2,3,4,6-tetrafluoro-5-iodobenzene and 2-(chloromethyl)1,3,4,5-tetrafluorobenzene to give calices 10, 11, 12, 13 and 14 respectively (Scheme 2). The use of different benzyl halides, which were easily accessible by Filkelstein substitution, was necessary together with adequate temperatures and reaction times to prevent dehalogenation. In most cases, the purification was conducted by column chromatography but an additional precipitation using methanol was necessary to avoid apparent ion transport effects due to solvent impurities.

\subsubsection{Ion transport}

There are a number of experimental approaches that permit measuring, or alternatively estimating, the ability of a given (macro)molecule to promote ion translocation across a lipid bilayer membrane [45]. Among these methods, the most convenient for initial screenings are 
experiments using large unilamellar vesicles (LUVs). The most common LUVs experiment is the HPTS assay, which directly indicates if a putative ion transporter shows transport activity in lipid bilayers. The main information obtained from this experiment is the effective molarity to obtain 50\% transport activity (EC50; the lower the value the higher the transport activity) and the Hill coefficient $(n)$ which gives an indication of the possible degree of aggregation needed to achieve transport. A number of variations of this experiment exist that further permit discerning between a symport and an antiport transport mechanism. We speak of a symport mechanism if both cations and anions are moved simultaneously across the membrane, and of an antiport mechanism if only cations or anions are exchanged though the membrane (Figure 4). Similarly, it is also possible to quantify the relative selectivity among different cations and anions. Other LUVs experiments have been developed that provide information on the membrane stability (e.g. CF assay that measures the formation of membrane defects leading to vesicle leakage as opposed to transport) or permit measuring directly the concentration of a given anion of interest (e.g. SPQ assay for chloride anions). These experiments can be combined to give a complete picture of the studied phenomena (please refer to Help Box 1 for further details).

Prior to observing any transport activity with the calix[4]arenes depicted in Scheme 2, a couple of unsuccessful attempts should be mentioned: the calix[4]arene derivatives still bearing the tert-butyl substituents (i.e. 7) and the use of cations other than tetramethylammonium (TMA+) during the transport experiments both where essentially inactive. When tetramethylammonium chloride (TMACl) was used in the HPTS (i.e. $200 \mathrm{mM}$ TMACl instead of a "standard" $100 \mathrm{mM}$ $\mathrm{NaCl}$ ), ion transport could finally be detected. Taken together, this indicates that the binding of TMA+, thus providing an additional ion-paring interaction, is necessary in this particular molecular system, to enable anion transport suggesting a symport mechanism [39]. It further suggests that transport is the result of a delicate equilibrium of non-covalent interactions.

However, the actual mechanism of transport was not immediately apparent. The first systems to be compared, calix $\mathbf{1 0}$ and $\mathbf{1 3}$, having respectively four perfluorophenyl or four meta-iodo perfluorophenyl arms gave confusing results: calix 10 had moderate activity $\left(E C_{50}=25 \mu \mathrm{M}\right)$ while calix 13 barely had any detectable activity. Following the same trend, the hydrogenbonding version of calix $\mathbf{1 3}$, in which the iodine was replaced by a hydrogen, also showed poorer transport activity $(E C 50=205 \mu \mathrm{M})$ than the fully perfluorinated calix $\mathbf{1 0}$. To rationalize these results, Matile and co-workers conducted NMR titrations to determine the binding affinity 
towards chloride of the studied calices, and performed density functional theory (DFT) calculations to both estimate the geometry and strength of the putative host-guest system.

The NMR titrations in deuterated acetone ([D6]acetone) using the bulkier and better soluble tetrabutylammonium chloride (TBACl) showed that only calix $\mathbf{1 3}$ could bind chloride strongly enough as to be detected under the experimental conditions $(K \mathrm{D}=18 \mathrm{mM}$ using a 1:1 binding isotherm). DFT molecular modeling at the PBE1PBE/6-311G** level of theory (Figure 1) provided the missing element: Calix $\mathbf{1 0}$ was shown to be able to form a stable complex with TMACl exploiting anion- $\pi$ interaction $(E=-58.3 \mathrm{kcal} \mathrm{mol-1})$ while calix 13 was able to form a much stronger complex by halogen bonding using only two out of four of the halogen bond donors $(E=-70.9 \mathrm{kcal}$ mol-1). The results of the HPTS assay, NMR titrations and DFT calculations were rationalized in term of a stronger binding inhibiting ion transport following the Goldilocks principle [46].

To confirm this hypothesis, the authors proposed two additional synthetic modifications: calix 11 bearing non-fluorinated iodophenyl substituents, and calix $\mathbf{1 2}$ in which a less favorable binding geometrical was introduced by switching from a meta to a para position on the perfluorinated iodophenyl unit. In both cases, a significant improvement in the measured transport activity $E C 50$ values was observed $\left(E C_{50}=32 \mu \mathrm{M}\right.$ and $62 \mu \mathrm{M}$ for calix 11 and 12 respectively). Additional measurements and DFT calculations further corroborated the proposed rational: Anion selectivity topologies (see Help Box 1 and Table 1) measured for calices 10 - 14 showed that the halogen-bond based systems had a marked preference for chloride over bromine and iodine, which is perfectly in line with the expected behavior of halogen bond donors. DFT calculations, on the other hand, confirmed that the estimated binding energies of the weaker receptor calix $12(E=-59.7 \mathrm{kcal} \mathrm{mol}-1)$ are similar to the anion- $\pi$ based calix $\mathbf{1 0}$ as it could be expected considering their similar transport activities.

The stoichiometry of binding and transport was also investigated [39]. Where NMR titrations were possible, a consistent 1:1 binding stoichiometry was observed both by the goodness of the fitting procedure, but also using Job plots. Similarly, a consistent Hill coefficient of about $n=$ 1 was measured for almost all calices. The exception for both the stoichiometries measured in solution (i.e. NMR titrations) and in the membrane (i.e. Hill coefficient as measured in the HPTS assay) was calix 12. While this is to be expected because of a less favorable geometry towards a focal cooperative binding, the measured stoichiometries did not agree between each other: 1:2 (calix to anion) as measured in solution and 2:1 in the membrane. Nevertheless, the global take away from this apparent inconsistency regarding calix 12 was that imposing 
unfavorable geometrical constraints leads to complex situations that cannot be rationalized in terms of simple unimolecular carrier transport systems.

\subsection{Oxacalix[2]arene[2]triazine}

The calix[4]arene macrocyclic systems were reported in 2011 [39], and no other macrocyclic system was published until 2019 [40] which can be understood as a testimony of the challenges involved in developing a suitable systems for transmembrane anion transport based on macrocyclic frameworks. The macrocyclic systems that will be discussed in the following were reported by the group of De-Xian Wang. They not only provide an additional example of an ion transport macrocyclic system having functional halogen bond donors, but further demonstrate that such systems can be used in vitro as anticancer agents, which represents another important step in the right direction.

\subsubsection{Synthesis}

The synthetic approach to preparing calices 18 - 21 was based on previous methodologies developed by the same group [47-50]. The one-pot approach employed was very attractive as it allowed them to prepare several candidates with moderate synthetic effort (Scheme 3). First, dichloro-substituted triazine $\mathbf{1 6}$ was prepared by nucleophilic substitution of cyanuric chloride 15 with the corresponding (di)bromo or (di)iodo phenol derivatives. Next, the one-pot reaction between a gallic acid ester-derivatized monobenzyl-protected diphenol with the triazine monomers 16 in presence of $\mathrm{K}_{2} \mathrm{CO}_{3}$ in refluxing acetonitrile gave the generic macrocyclic compound 17. The straightforward deprotection of the benzyl group using $\mathrm{AlCl}_{3}$ in dry toluene afforded calices $\mathbf{1 8}-\mathbf{2 1}$ in very good overall yields (14.8\% - 25.7\% over three steps). The control calix 22 was prepared following a different procedure [40].

\subsubsection{Anion binding studies}

The authors evaluated first the chloride and bromide binding affinity of calices $\mathbf{1 8}-\mathbf{2 1}$ through NMR titrations in deuterated acetone [D6]acetone using TBACl and $\mathrm{TBABr}$. They chose to follow the changes in chemical shift of the hydroxy proton signals as it was larger than the, still detectable, shift of the resonances on the halogen-substituted phenyl groups. The fact that the putative 1:1 binding observed affects the hydroxy protons as well as the halogen-bond donor units indicates that a cooperative effect is probably taking place.

The binding affinities measured for chloride were moderate $\left(\mathrm{K}_{\mathrm{a}}=7.37 \times 10_{3}-1.30 \times 10_{4}\right.$ for $\mathbf{1 8}-\mathbf{2 1}$ respectively) as were the ones for bromide $\left(K_{a}=6.34 \times 10_{2}-1.00 \times 10_{3}\right.$ for $\mathbf{1 8}-\mathbf{2 1}$ 
respectively) with a clearly weaker overall binding affinity for bromide as expected for a halogen-bond donor receptor. To clarify the cooperative effect of various non-covalent interactions, crystallographic studies were carried out next.

\subsubsection{Crystallographic studies}

Crystals of sufficient quality were obtained for the complexes of calices 19 and 20 with chloride. The crystal structures not only showed the presence of strong hydrogen bonds and short contacts attributed to halogen bonding with the chloride anion, but also significant influence of anion- $\pi$ interactions. Indeed, the structures elucidated are rich in information: For complex $19 \cdot \mathrm{Cl}$ - a strong competition among multiple non-covalent interactions is made apparent with a marked preference to form intermolecular interaction using the chloride anion as bridge. The crystal structure of complex $\mathbf{2 0} \cdot \mathrm{Cl}$ - (see Figure 2 ) allowed the authors to differentiate two types of capsules formed by the positioning of the iodines of a single given calix in either a syn or an anti configuration. As it was the case for $19 \cdot \mathrm{Cl}_{-}$, the chloride anion was also involved in intermolecular interactions creating an infinite network of interpenetrated capsules. In both cases, the synergetic effect of hydrogen and halogen bonding together with additional anion- $\pi$ interactions was observed.

\subsubsection{Ion transport}

To probe anion transport using calices $\mathbf{1 8}$ - 21, a number of experiments in LUVs were conducted. First, all compounds were tested in the Lucigenin assay (see Help Box 1) and showed transport activities in the range of $E C 50=7.4 \mu \mathrm{M}$ and $E C_{50}=3.1 \mu \mathrm{M}$ when using $\mathrm{NaCl}$ $(100 \mathrm{mM})$ as salt. Exchanging the cation had only minor effects over the alkaline series and the exchange of the anion (i.e. using $\mathrm{NaBr}$ ) showed a minor, yet consisted, decrease in activity for all calices $18-\mathbf{2 1}$.

Additional experiments using fluorogenic LUVs, such as the acceleration of proton exchange by the addition of carbonyl cyanide-4-(trifluoromethoxy)phenylhydrazone (FCCP) or the acceleration of potassium exchange by the addition of valinomycin in the HPTS assay [45], suggest that transport proceeds following an antiport mechanism.

Calix 22 was used as a control experiment because this calix is not able to form halogen bonds. Curiously, when this compound was tested in the Lucigenin assay, a marginally higher ion transport activity was recorded $\left(E C_{50}=2.2 \mu \mathrm{M}\right)$ in the HPTS assay. While this result indicates that halogen bonding is not crucial for observing ion transport, the ion selectivity observed for the series 18 - 21 probably benefits from the high specificity of halogen bonding. On the other 
hand, if the free phenols on the macrocycle are blocked (by ether formation), the described oxacalix[2]-arene[2]triazine systems becomes entirely inactive [40].

In sharp contrast with the calix[4]arene case discussed before, the ion transport enabled by the oxacalix[2]-arene[2] triazine family (18 - 21) was characterized by Hill coefficients consistently above one (see Table 1). This suggests, because a clear interpretation of the Hill coefficient is difficult [45], that complex dimers and trimers are the active transport species.

\subsubsection{Anticancer activity}

Having demonstrated that calices $\mathbf{1 8}-\mathbf{2 1}$ could promote ion transport in model LUVs, the authors decided to test if such functional property could also be employed in vitro notably towards treatment of cancer cells. The study was carried out using the colorectal carcinoma cell line HCT116. They observed that all active ion transporters significantly reduced the cell viability $(I C 50=52.3,58.9,66.4$, and $54.8 \mu \mathrm{M}$ for calices $\mathbf{1 8}-\mathbf{2 1}$ respectively). While control calix 22 was not tested, it was shown that the presence of hydroxy groups on the calix was also crucial for the anticancer activity.

Table 1. Summary of transport and binding data for macrocyclic systems [a].

\begin{tabular}{ccccccc}
\hline & Calix & $E C 50 / \mu \mathrm{M}[\mathrm{b}]$ & $n[\mathrm{c}]$ & $\mathrm{Cl} / \mathrm{Br}-[\mathrm{d}]$ & $\mathrm{NO} 3-/ \mathrm{AcO}-[\mathrm{d}] \mathrm{KD} / \mathrm{mM}[\mathrm{e}]$ \\
\hline 1 & $\mathbf{1 0}$ & $25 \pm 5[\mathrm{f}]$ & $1.1 \pm 0.2$ & 1.1 & 2.1 & n.d. \\
5 & $\mathbf{1 1}$ & $32 \pm 15[\mathrm{f}]$ & $1.4 \pm 0.8$ & 2.8 & 1.5 & n.d. \\
4 & $\mathbf{1 2}$ & $62 \pm 8[\mathrm{f}]$ & $1.9 \pm 0.5$ & n.d. & n.d. & 13.3 \\
2 & $\mathbf{1 3}$ & $\sim 1000[\mathrm{f}]$ & $0.7 \pm 0.3$ & 3.4 & 1.7 & 18.0 \\
3 & $\mathbf{1 4}$ & $205 \pm 6[\mathrm{f}]$ & $1.04 \pm 0.03$ & 1.5 & 1.8 & n.d. \\
6 & $\mathbf{1 8}$ & $7.4 \pm 0.5$ & $3.2 \pm 0.7$ & 1.4 & n.d. & 0.14 \\
6 & $\mathbf{1 9}$ & $5.5 \pm 0.1$ & $4.1 \pm 0.4$ & 1.3 & n.d. & 0.11 \\
6 & $\mathbf{2 0}$ & $4.0 \pm 0.2$ & $3.2 \pm 0.6$ & 1.7 & n.d. & 0.03 \\
6 & $\mathbf{2 1}$ & $3.1 \pm 0.1$ & $2.2 \pm 0.2$ & 0.9 & n.d. & 0.07 \\
6 & $\mathbf{2 2}$ & $2.2 \pm 0.7$ & $1.3 \pm 0.7$ & n.d. & n.d. & n.d. \\
\hline
\end{tabular}

[a] For the original data, see reference [39] and [40]. [b] Effective calix concentration needed to reach 50\% activity in the HPTS assay. [c] Hill coefficient. [d] Relative calix activity in the HPTS assay with different extravesicular anions. [e] Dissociation constant 


\section{Help Box 1: Ion Transport experiments in Large Unilamellar Vesicles (LUVs)}

A vesicle is a rather convenient model of the cellular membrane because the composition of the membrane can be tuned to be as close as necessary to the real system. On the other hand, since it is an artificial system, its composition is more stable and reproducible and, thus, a more robust alternative. It also has the advantage to require little specific equipment making it a widely accessible method. The main challenge in employing vesicles, and in particular of LUVs, to quantify ion transport is the need to quantify either very weak changes in concentration outside of the vesicles, or to probe the inside of said vesicles. A few approaches have been proposed over the last 20 years including NMR [41,52] and ion selective electrodes [53,54], but the arguably most successful approach uses fluorescent indicators encapsulated inside the vesicles [45]. The nature of the fluorescent dye inside the vesicle will give information on specific changes.

The most common experiment is the HPTS assay. In this assay, vesicles containing 8hydroxy-1,3,6-pyrenetrisulfonate (HPTS, Figure 3) are prepared and then suspended in a solution of comparable osmotic pressure. HPTS being a pH-sensitive fluorophore, the $\mathrm{pH}$ at the interior of the vesicles can be followed by simply measuring the fluorescence using a standard spectrofluorometer. Since vesicles are closed systems, a $\mathrm{pH}$ change outside the vesicles will have no significant effect on the fluorescence signal. If, on the other hand, a (macro)molecule able to promote the translocation of ions through the membrane is trapped in the membrane, the transport of anions or cations entails a change in $\mathrm{pH}$ that ultimately leads to the equilibration of the $\mathrm{pHs}$ inside and outside of the membrane. The natural consequence is that most ion transporters, regardless their specificity or mechanism, can be studied using this assay.

From a practical point of view, a change in $\mathrm{pH}$ is induced outside the vesicles by the addition of a base (typically $\mathrm{NaOH}$ ), which is followed by the transporter at a given concentration. At the end of the experiment, the $\mathrm{pH}$ is equilibrated by the addition of a detergent which disrupts the membrane. The concentration of transporter is tuned in 
such a way as to permit the observation of the global transport kinetics on a macroscopic time-scale.

By repeating the measurement at several concentrations, it is possible to construct the so-called dose-response curve which shows the fractional transport activity as a function of the concentration. Given a number of assumptions [45], this curve can be fitted to the Hill equation (Eq. 1) in which $Y$ is the fractional transport activity, $c_{M}$ the concentration of the transporter, $Y_{\mathrm{MAX}}$ the maximal detectable activity, $Y_{\mathrm{MIN}}$ the activity in absence of transporters, $E C 50$ the monomer concentration needed to achieve $50 \%$ of transport activity, and $n$ the Hill coefficient which can describe the number of monomers needed to observe activity [55-57].

$$
Y=Y_{M A X}+\frac{\left(Y_{M I N}-Y_{M A X}\right)}{\left(1+\frac{C_{M}}{E C_{50}}\right)^{n}}
$$

Provided the same conditions are employed, the $E C_{50}$ and $n$ values are crucial to compare different transport systems.

Using HPTS as fluorescent indicator is convenient because recording the fluorescent intensity at two different wavelengths, which correspond to the maxima of the neutral and protonated forms, permits measuring the change in $\mathrm{pH}$ and avoids issues related to single intensity measurements. Yet, HPTS does not report on the concentration of a specific anion or cation. Issues of specificity can be circumvented by the use of ion specific fluorophores such as Lucigenin [58] or 6-methoxy-N-(3sulfopropyl)quinolinium (SPQ) [59] and by applying the same principles to the data analysis. In such case, the respective experiments would be the Lucigenin and SPQ assays.

With these assays and a few clever variations that help discerning transport mechanisms (antiport vs symport) [45], a good characterization of ion transport systems is possible using only minor technical resources. One last question, though, needs also be addressed and it is the formation, induced by the added (macro)molecules, of membrane imperfections that temporary permit exchange or leaks. To interrogate about this possibility, another variation of LUVs experiment can be used: the CF assay. In this assay, a self-quenched concentration of 
carboxyfluorescein (CF) is encapsulated in LUVs. In absence of membrane defects, $\mathrm{CF}$ remains inside the vesicles and shows only low fluorescence; if the added (macro)molecules, or the experimental conditions, lead to membrane defects, CF escapes the vesicles and an increase in fluorescence is observed. In a typical example, both the results of the HPTS and CF assays will be fitted to the Hill equation (Eq. 1) and a comparison of the obtained $E C 50$ s (for transport and leakage respectively) will allow to avoid the misinterpretation of the HPTS results. Every halogen-bond based ion transporter described in this chapter was consistently tested in the CF assay and the absence of major defects in the membrane was therefore confirmed.

\section{Ion Selectivity}

One additional feature of the HPTS assay is the possibility to directly compare the relative rates of ion transport as a function of the anion and/or cation involved. Naturally, any ion transport system will be more efficient for a given ion, or ion pair, according to the mechanism of ion translocation and the non-covalent forces involved. For instance, a (macro)molecule that operates by a clean anion antiport mechanism (Figure 4), will not be affected by the presence (or absence) of cations but will show large differences if the anion being transported is exchanged for a difference one. The latter situation depends on the degree of selectivity which in turn is related to, but not determined by, the binding affinity to the anion. This indirect relationship is often considered as a good indication of a putative ion transporter, but has also been shown to fail in cases where the "Goldilocks principle" prevails (i.e. ion transport can be inhibited by binding too strongly and leading to insufficient release) [46].

Practically, the ion selectivity can be investigated in the HPTS assay by exchanging the external ion making it is possible to easily address several cations and anions. The results of these studies are typically presented in selectivity topologies in which the normalized fractional transport activity is presented as a function of the hydration energy of the ion (Figure 4e,f). In such a representation, it is easy to identify if the transport activity is directly related to the hydration energy or if the binding energy to the ion overcompensates this sequence. These ion selectivity topologies are known as Hofmeister or anti-Hofmeister respectively in honor to Hofmeister and his work on protein denaturation more than a century ago [60]. 


\section{Small molecules}

It is apparent from the previous examples on macrocyclic systems that developing multitopic transmembrane ion transporters is challenging, possibly due to issues related to the larger van der Waals radius and the intrinsic high directionality of halogen bonding. This was also shown for research on halogen-bond receptors where the natural binding preference towards anions can be significantly influenced - or even inverted - when multitopic systems are concerned $[34,36,61]$. Moreover, the association constants measured for the calix[4]arene systems were comparable to the association constants reported, and measured, for simpler monotopic systems. Motivated by these observations, the study of comparatively simpler molecular systems was proposed [24,51].

\subsection{Iodo-perfluoro arenes}

The natural first choice were iodo-perfluoro arenes because this family of compounds can be seen as a simplification of the calix[4]arene system. The authors decided to use commercially available molecules and the series $\mathbf{2 3}$ - 26 (Scheme 3) was investigated for ion transport activity using the HPTS assay. All three iodo arenes were active with $E C_{50}$ values close to the ones measured for the best calix[4] arene systems $\left(E C_{50}=260 \mu \mathrm{M}, 67 \mu \mathrm{M}\right.$ and $26 \mu \mathrm{M}$ for 23, 24 and 25 respectively). Although the measuring conditions differed on the buffer composition (i.e. $200 \mathrm{mM}$ TMAC, $10 \mathrm{mM}$ HEPES, $\mathrm{pH}=7$ for 11 to 14 and $100 \mathrm{mM} \mathrm{NaCl}, 10 \mathrm{mM}$ HEPES, $\mathrm{pH}$ $=7$ for $\mathbf{2 3}-\mathbf{3 7}$ ), this was a remarkable result considering the simplicity of the tested molecules. Bromo-perfluoro benzene 26 was almost completely inactive in the HPTS assay $\left(E E_{50}=1.9\right.$ $\mathrm{mM})$ as was the non-fluorinated iodo-benzene $37\left(E C_{50} \sim 2 \mathrm{mM}\right)$ as expected for a system functioning mainly based on halogen bonding (i.e. better binding leading to better transport activity) [51]. This was further confirmed by ion selectivity measurements showing, for compounds $23-\mathbf{2 5}$, only negligible differences in transport activity by exchanging the cation in the HPTS assay, and major changes in transport activity as result of an exchange of the anion $\left(\mathrm{Cl}_{-}>\mathrm{Br}->\mathrm{I}_{-}\right)$. Two additional control experiments were presented: Perfluoro benzene 35, which had no detectable activity in the HPTS assay, suggested that anion- $\pi$ interactions could not promote ion transport by itself; and a strong, but highly hydrophilic, halogen-bond donor 36, which was also inactive in the HPTS assay, demonstrated that strong binding is not sufficient for halogen-bonding driven ion transport.

Table 2. Summary of transport activity for small molecules [a]. 


\begin{tabular}{cccccc}
\hline Arene & $E C 50 / \mu \mathrm{M}[\mathrm{b}]$ & $n[\mathrm{c}]$ & Alkane & $E C 50 / \mu \mathrm{M}[\mathrm{b}]$ & $n[\mathrm{c}]$ \\
\hline $\mathbf{2 3}$ & $260 \pm 10$ & $3.6 \pm 0.4$ & $\mathbf{2 7}$ & $>2000$ & n.d. \\
$\mathbf{2 4}$ & $67 \pm 4$ & $2.1 \pm 0.2$ & $\mathbf{2 8}$ & $3.07 \pm 0.15$ & $3.3 \pm 0.4$ \\
$\mathbf{2 5}$ & $26 \pm 1$ & $2.3 \pm 0.2$ & $\mathbf{2 9}$ & $21.6 \pm 0.6$ & $4.7 \pm 0.6$ \\
$\mathbf{2 6}$ & $\sim 2000$ & n.d. & $\mathbf{3 0}$ & $85 \pm 4$ & $2.6 \pm 0.3$ \\
$\mathbf{3 5}$ & n.a. & n.d. & $\mathbf{3 1}$ & $30.0 \pm 0.9$ & $3.4 \pm 0.4$ \\
$\mathbf{3 6}$ & n.a. & n.d. & $\mathbf{3 2}$ & $1450 \pm 90$ & $2.9 \pm 0.4$ \\
$\mathbf{3 7}$ & $\sim 2000$ & n.d. & $\mathbf{3 3}$ & n.a. & n.d. \\
& & & $\mathbf{3 4}$ & n.a. & n.d. \\
\hline
\end{tabular}

[a] For the original data, see reference [51]. [b] Effective concentration needed to reach $50 \%$ activity in the HPTS assay (100 mM NaCl, $10 \mathrm{mM}$ HEPES, $\mathrm{pH}=7)$. [c] Hill coefficient. n.d.: not determined. n.a.: no transport activity detected.

\subsection{Iodo-perfluoro alkanes}

The remarkable results obtained with the iodo-perfluoro arenes were used as starting point to further simplify the system. Towards this goal, the use of iodo-perfluoro alkanes was envisioned to completely exclude any contribution from anion- $\pi$ interactions. Here as well, commercially available compounds were considered first, and a collection having different lengths of the carbon chain was investigated (27 - 32; Scheme 4).

The results obtained in the HPTS assay could not have been better: Not only these compounds - of astonishing structural simplicity - gave detectable ion transport activities, but the best transport activity observed for this series was almost an order of magnitude better than their arene counterpart (e.g. $E C_{50}=26 \mu \mathrm{M}$ for $\mathbf{1 6}$ and $E C 50=3.07 \mu \mathrm{M}$ for 28). Moreover, when considering the complete iodo-perfluoro alkane series, a clear trend is apparent which shows the best transport activity for the 1-iodo-perfluoro hexane $\mathbf{2 8}$, and gradually decreasing transport activities when either increasing or decreasing the chain length (Figure 6a).

From the above-described series, trifluoroiodomethane $\mathbf{3 2}$ is a somehow particular case: Unlike its non-fluorinated counterpart, compound 32 is a gas at room temperature (b.p. $=-22{ }^{\circ} \mathrm{C}$ ) and could also be delivered to fluorogenic LUVs by bubbling the compound through the suspension 
of LUVs to promote ion transport. Unsurprisingly, its transport activity can at best be qualified as poor $\left(E C_{50}=1450 \mu \mathrm{M}\right)$, but given its uniquely simple structure (i.e. five atoms in total and a single carbon), its natural occurrence as a gas, and the unlikeliness of a simpler organic molecule fulfill such a non-trivial task, this compound is to be considered the smallest organic anion transporter ever. This beautifully illustrates the inherent compatibility of halogen bonding and transmembrane anion transport which can be attributed to the formation of strong non-covalent bonds with anions while employing an essentially hydrophobic molecular framework. It is important to mention that transmembrane anion transport has been reported in the past using molecular iodine and bromine [62,63], which proceeds through the formal formation of the corresponding $\mathrm{I}_{3}^{-}$and $\mathrm{IBr}_{2}^{-}$and other higher species. Since it is unclear if such a complex can be considered a halogen bonded species, and the experimental difficulties of measuring transmembrane anion transport with a highly oxidizing compound, we have chosen not to include a more detailed description.

As before, the ion selectivity measurements in the HPTS assay showed a clear anion dependence of the measured transport activity. Furthermore, control experiments using perfluorohexane $\mathbf{3 3}$ or 1-iodo hexane $\mathbf{3 4}$, showing no detectable ion transport, further confirm the crucial role of halogen bonding to promote anion transport. The formation of membrane defects could also be excluded by consistently testing the membrane stability using the CF assay.

To confirm the results in the HPTS assay, and further characterize these minimalistic anion transporters, the authors resorted to conductance experiments in planar lipid bilayer membranes which will be discussed in the following section. A short description of this technique can be found in Help Box 2, and for further information the reader is referred to specialized literature $[7,45]$.

Help Box 2: Conductance experiments in planar lipid bilayer membranes

Conductance experiments in planar lipid bilayer membranes, also called Black Lipid Membrane (BLM), is another alternative to characterize ion transport activity [45]. While both techniques, LUVs and BLMs, can be used to assess ion transport in lipid bilayer membranes, there are specific cases that require the use of one of them 
specifically. The quintessential example where conductance experiments are required is the characterization of ion channels and pores. These measurements represent the clearest - if not the only - conclusive evidence of a channel ion transport mechanism. Nevertheless, the need of specific equipment to carry out conductance experiment in planar bilayers often hampers its wider use.

Experimentally, this technique consists of two chambers separated by a small hole of typically $50-200 \mu \mathrm{m}$ in diameter. These chambers, named cis and trans for historical reasons, are filled with a buffer (which acts as electrolyte) and connected to the measuring electrodes through salt bridges. The small connecting hole is covered by a single lipid bilayer membrane, which can be deposited by various different approaches $[64,65]$, that will act as an insulating barrier. The capacitance measured can further be used to characterize the lipid bilayer membrane prior to any transport experiments [7]. A schematic representation of the experimental set-up is depicted in Figure 5.

In absence of ion transporters or membrane defects, no current will be detected because the lipid bilayer membrane acts as an insulator. If a (macro)molecule having ion transport activity is present in the membrane, a small current will be detected and is directly related to the transport of ions from one chamber to the other. In contrast to ion transport experiments in LUVs, conductance measurements have much better sensitivities and temporal resolution $[7,16]$. This is particularly important when studying channels and pores and aiming at capturing single molecular events [66].

Two main cases can be distinguished: ion channels and pores, and ion carriers. When investigating the transport activity of ion channels and pores in conductance experiments, a sharp step-like increase in current is expected. Such events are the direct observation of the ion transport through the channel or pore and can be described by its characteristic conductance $g$, lifetime $\tau_{1}$, and open probability $\mathrm{P}_{0}$. As seen in Figure 5b, channels and pores intercalate between open and closed events, and it is not uncommon to observe multi-step events. From the afore mentioned parameters, it is possible to estimate the channel/pore size using the Hille equation (Eq. 2), in which $\rho$ is the resistivity of the electrolyte, $g$ is the conductance, $l$ the length, and $r$ the radius of the putative channel or pore $[7,45]$. 


$$
\frac{1}{g}=\left(l+\frac{\pi \cdot r}{2}\right) \cdot\left(\frac{\rho}{\pi \cdot r^{2}}\right)
$$

Ion carriers, on the other hand, will not show such a step-like behavior but will be characterized by a gradual increase of the measured current until it reaches a saturation value that characterizes the putative ion carrier under those conditions (Figure 5c).

Up to this point, we have only considered symmetric conditions (cis and trans chambers containing the same electrolyte at the same concentrations) and we have only considered the same applied voltage. These parameters can be readily modified to investigate ion selectivity and permeability, and voltage dependence among numerous other parameters that are discussed elsewhere $[7,45]$.

\section{Ion selectivity}

The very nature of the conductance experiments in bilayer membranes entails that the direction of the flow of charges is known: the input electrode is connected to the cis chamber and the reference electrode to the trans chamber. The consequence is that if an asymmetric gradient of concentrations is employed between the cis and trans chambers, it is possible to determine directly if the ion transporter is selective for cations or anions and to which extent this preference is respected. An example of such a measurement is depicted in Figure 5e were a concentration gradient of $2 \mathrm{M}$ against $0.25 \mathrm{M}$ (cis to trans) is used during the measurement. Because the applied voltage needs to compensate the imposed ion gradient, a non-zero voltage needs to be applied to observe a zero current. The voltage required to stop the ion flow induced by a difference in concentrations is called the reverse potential $V_{\mathrm{r}}$. If $V_{\mathrm{r}}$ is positive, the system is selective towards the transport of anions, and if $V_{r}$ is negative, we speak of a cation selective transport. Moreover, beyond determining cation and anion selectivity, it is also possible to quantify the degree of selectivity, also called permeability ratio, by applying the Goldman-Hodgkin-Katz (GHK) voltage equation (Eq. 3):

$$
\frac{P_{A^{-}}}{P_{M^{+}}}=\frac{a_{M} \text { cis }-a_{M} \text { trans } \cdot \exp \left(\frac{-V_{r}}{R T}\right)}{a_{A} \operatorname{cis} \cdot \exp \left(\frac{-V_{r}}{R T}\right)-a_{A} \text { trans }}
$$


Where $\frac{P_{A^{-}}}{P_{M^{+}}}$is the anion/cation permeability ratio; $a_{M}$ cis the cation activity in the cis chamber, $a_{A}$ trans the anion activity in the trans chamber and so on; $R$ is the gas constant; $F$ is the Faraday constant; $V_{r}$ is the reverse potential.

\section{Voltage gating}

Another straightforward option when conducting conductance experiments in bilayer membranes is to measure the transport activity as a function of the applied voltage. Here, the trivial case gives a linear dependence of the observed current $I$ with the applied voltage $V$ following Ohm's law (Eq. 4).

$$
I=g \cdot V
$$

Eq. 4

Nevertheless, many examples, either from nature or artificial, do not follow Ohm's law and are called voltage dependent channels, pores and carriers [32,67]. Such systems can be easily identified (e.g. Figure 5d), and the extent and nature of this voltage dependence is best characterized by its gating charge $z \mathrm{~g}$ defined by the following equation (Eq. 5):

$$
Y \propto g \propto c_{M}^{n} \cdot \exp \left(\frac{z_{g} \cdot e \cdot V}{k \cdot T}\right)
$$

Where $\mathrm{Y}$ is the fractional activity; $c_{M}$ is the monomer concentration; $n$ is the Hill coefficient; $e$ is the elementary charge; $k$ is the Boltzmann constant; and $T$ is the absolute temperature. It is worth mentioning that it is also possible to estimate the gating charge in LUVs by creating a membrane potential as described elsewhere [45].

\subsection{Conductance experiments in planar lipid bilayers with small molecules}

In conductance experiments in lipid bilayer membranes (also known by their abbreviation BLM), the transport activity of any given (macro)molecular system is observed as a change in the measured current $I$, at a given applied voltage, between two chambers separated by a flat lipid bilayer (see Help Box 2). Given that these measurements require significantly more time to be completed than LUVs experiments, the authors focused their attention to compounds $\mathbf{2 3}$, 24, and 29. In all three cases, a steady increase in current was observed following the addition 
of the putative transporter to the buffer solution in the cis chamber. Consistently, a saturation value in current was also observed corresponding to the characteristic activity of the given transporter at the measured conditions (buffer, transporter concentrations and applied voltage). A typical trace for iodo-perfluorobenzene $\mathbf{2 3}$ is presented in Figure 5c. On the one hand, the measurement of an increase in current in presence of the putative transporters strongly supports the results from the HPTS assay confirming that the tested molecules can act as transmembrane ion transporters. On the other hand, the shape of the BLM trace is characteristic for a carrier mechanism, which is reasonable considering the small size of the compounds.

The dependence of the observed ion transport on the applied voltage was investigated next. A strongly non-linear dependence was observed for all the investigated compounds (e.g. Figure 5d). This non-ohmic behavior was characterized by gating charges up to $z \mathrm{~g}=0.41$ for iodoperfluorobenzene $\mathbf{2 3}$. Increasingly high gating charges are typically observed in biological ion channels with specialized gating mechanisms, but have also been observed for artificial ion channels [69]. For an ion transporter that operates under a carrier mechanism, such a high value is significant and is typically observed for highly selective carriers. It suggests that association and dissociation at the membrane interface are fast compared to the translocation of the loaded carrier across the membrane, meaning that at higher voltages a direct acceleration of this ratelimiting process takes place.

The ion selectivity of these minimalistic ion anion transporters was also investigated. As opposed to LUVs experiments which can only qualitatively measure ion selectivity, BLM measurements permit to precisely quantify the extent of said selectivity based on the shift of the zero-current voltage also called reverse potential (Figure 5e and Help Box 2). For the three

studied compounds $\left(\mathbf{2 3}, \mathbf{2 4}\right.$ and 29), anion/cation permeability values as high as $\frac{\mathrm{P}_{\mathrm{Cl}^{-}}}{\mathrm{P}_{\mathrm{K}^{+}}}=37$ (for 29) were obtained. A clear difference could be observed between the arene and alkane compounds, with the former performing less well at anion/cation permeability ratios below $\frac{\mathrm{P}_{\mathrm{Cl}^{-}}}{\mathrm{P}_{\mathrm{K}^{+}}}$ $=11$. The high selectivity of these three compounds demonstrates a clean anion antiport mechanism.

\subsection{Elucidating the mechanism of transport with small molecules}

Based on the described experiments, it was established beyond any reasonable doubt that the studied halogen bond donors could act as anion carriers with good transport activities. But the 
mechanism behind this activity and the nature of the active species was still not understood. To gain insights on the molecular basis of the observed anion transport activity, four main resources were employed: Further LUVs experiments to confirm a carrier mechanism; an analysis of the measured Hill coefficients across the series; DFT molecular modeling of plausible structures, and experimentally address the likeliness of the hypothetical structures by solid-state crystal engineering.

\subsubsection{Membrane composition in the HPTS assay}

For all the LUVs experiments describe so far, egg yolk phosphatidylcholine (EYPC) was used to prepare the unilamellar vesicles. Using this lipid, which is formally a zwitterion at $\mathrm{pH}=7$, results in a mildly positively-charged, or even completely neutral, vesicle surface. If egg yolk phosphatidylglycerol (EYPG) is used instead, this negatively charged lipid results in a negatively charged surface. All the reported small molecules $(\mathbf{2 3}-\mathbf{3 2})$ were also tested in the HPTS assay using EYPG systematically showing a decrease in ion transport activity of almost an order of magnitude [51]. This result indicates that the transport mechanism needs to involve negatively charged species that will be repelled by the membrane hampering the transport activity.

In a similar manner, in another set of experiments, EYPC was replaced by dipalmitoyl phosphatidylcholine (DPPC). The aim on this experiment was to investigate the influence of the membrane fluidity on the observed ion transport activity. Indeed, in contrast to EYPC that displays a constant fluid phase $\mathrm{L}_{\alpha}$ in the range $5-50{ }^{\circ} \mathrm{C}$, DPPC has the following phase (pre)transitions in the same range: pseudocrystalline $\mathrm{L}_{\mathrm{c}^{\prime}}$ into the crystalline gel phase $\mathrm{L}_{\beta^{\prime}}$ at $\mathrm{T}$ $=19{ }^{\circ} \mathrm{C}$, and the main transition from the ripple phase $\mathrm{P}_{\beta^{\prime}}$ into the fluid phase $\mathrm{L}_{\alpha}$ at $\mathrm{T}=41{ }^{\circ} \mathrm{C}$. The ion transport activity of $\mathbf{2 3}, \mathbf{2 5}$, and $\mathbf{2 9}$ was measured in the HPTS assay using DPPC LUVs at various temperatures in the range $15-50{ }^{\circ} \mathrm{C}$. All three compounds showed a significant decrease in activity in the less fluid phases $\left(<20^{\circ} \mathrm{C}\right)$ with difference as high as 30 -fold higher activity when operating in a fluid phase. While the arene compounds $\mathbf{2 3}$ and $\mathbf{2 5}$ showed a constant and gradual increase in activity with increasing temperature, the alkane transporter 29 also showed big changes in transport activity around the afore mentioned phase (pre)transitions (Figure 6b) implying a more pronounced influence of the fluidity of the membrane. In all cases, a strong dependence on the membrane fluidity was interpreted as evidence of a carrier mechanism. 


\subsubsection{Hill coefficients}

We have already introduced the Hill coefficient and the ways to interpret it when discussing the macrocyclic compounds. In those cases, the measured Hill coefficients were mostly equal to one in line with the predicted 1:1 complexes which have been proposed as active transport structures. The case of the iodo-perfluoro arenes and alkanes $(\mathbf{2 3}-\mathbf{3 2})$ shows the opposite: every single compound tested gave Hill coefficients above $n=2.1$, with iodo-perfluorobutane 29 being the highest at $n=4$.7. The interpretation of Hill coefficients is not trivial, but such a consistent occurrence of high Hill coefficients can safely be interpreted as evidence of a multimolecular active species being responsible for the ion transport activity. Based on these observations, the authors proposed a self-assembled structure involving multiple halogen bond to a central anion (Figure 6c). The likeliness of such a structure was then investigated by molecular modeling and crystal engineering.

\subsubsection{Molecular modeling: DFT calculations}

The proposed superstructure was studied by DFT using the M06-L/6-311G** level of theory for geometry optimizations and M06-2X/6-311G** level of theory for binding calculations. The authors used iodo-perfluorobutane $\mathbf{2 9}$ as the model halogen-bond donor and chloride as the anion. With increasing number of binders, from one to six, an increase in binding energy was evidenced in gas-phase calculations. The fully optimized structure for the complex $6 \times \mathbf{2 9} \cdot \mathrm{Cl}-$ gave an almost perfectly octahedral geometry with a corresponding interaction energy of $E=-$ $90.1 \mathrm{kcal} \mathrm{mol-1}$. A similar procedure but using the hydroxy anion was also successful, but only up to five halogen bond donors $\mathbf{2 9}$ could be accommodated due to the presence of a hydrogen atom (i.e. $5 \times \mathbf{2 9} \cdot \mathrm{OH}-$ ). In this case, the calculated interaction energy was $E=-112.2 \mathrm{kcal}$ mol1. This suggested that both complexes are favorable and could account for the shuttling of both chloride and hydroxy anions.

\subsubsection{Crystal structures}

Because it is almost impossible to study supramolecular complexes inside lipid bilayers, the authors decided to use crystal engineering to explore experimentally the likeliness of the proposed structure. Ternary co-crystals were prepared using crown ether 18 -crown-6, $\mathrm{KCl}$, and iodo-perfluorobutane 29. As shown in Figure 2, the X-ray structure that was obtained clearly 
shows two halogen-bond donors $\mathbf{2 9}$ binding simultaneously to the chloride anion. Unfortunately, the presence of 18-crown-6 hinders any additional binding to the anion. Nevertheless, the formed structure at a geometry close as the one predicted (i.e. halogen bonding at approximately $180^{\circ}$ with respect to each other) was interpreted as evidence of that family of structures.

The overall conclusion suggests that a molecular arrangement similar to the one depicted in Figure $\mathbf{6 c}$ is likely responsible for the anion transport observed. Concerning the mechanism of the process, it was demonstrated that anion transport proceeds following a remarkably clear and selective anion antiport transmembrane mechanism. Finally, the almost absurd simplicity of the molecules involved leaves no room to any other explanation to explain the observed anion translocation other than it being promoted by halogen bonding, and thus the suitability of this non-covalent interaction for this particular application.

\section{Halogen bonding ion channels}

Based on the results obtained with the minimalistic halogen bond donors when applied to anion transport in bilayer membranes, a few challenges remained to be accomplished: To prepare artificial equivalents to ion channels, to improve the anion transport activity, and ultimately to apply the learned lessons towards their application in, for instance, drug discovery. In the following, we will discuss two examples of ion transport systems that are likely to function following a mechanism similar to ion channels. The first one, published in 2013 by Matile and co-workers [70], explores a covalent approach to prepare a rigid molecule that can promote anion transport by a putative hopping mechanism among halogen-bond donors. The second example, published in 2018 by Zeng and co-workers [68], explores the use of supramolecular chemistry not only for the binding and release of the transported anion, but also to assemble the backbone of the ion channel in a beautiful illustration of the orthogonal application of hydrogen and halogen bonding.

\subsection{Halogen bonding cascades}

Following a similar logic to the one described for macrocyclic systems, the development of ion channels using halogen bonding was based on an existing and well-studied scaffold: The $p$ oligophenyls rigid rods which have been extensively studied with the complementary hydrogen bonding interaction [14,71,72]. From those studies, crucial information regarding the positioning of the rigid rod in the membrane and its mechanism of action, at least for the 
hydrogen bonding case, are known. The global idea was to adapt this system by introducing eight flexible arms capable of forming halogen bonds. Here again, the use of iodoperfluorophenyl substituents was considered to be the best option. Because of the results previously obtained with the macrocyclic systems, it was considered necessary to use perfluorophenyl analogues as control experiments to evaluate the effect of anion- $\pi$ interactions.

\subsubsection{Synthesis}

The synthesis of the $p$-oligophenyl rigid rod of increasing oligomeric length has already been described in the literature [14]. Following these procedures, it was possible to prepare the $t \mathrm{Bu}-$ protected carboxylic acid version of the bi-, quarter-, sexi- and octiphenyl rigid rods $(39-\mathbf{4 2})$ starting from commercially available Fast Blue salt 38. Deprotection of the tert-butyl protecting group gave access to the free acid analogues $\mathbf{4 3}-\mathbf{4 6}$. The final coupling using fluorinated benzyl bromide (9) for compounds 51 - 54, and 4-iodo 2,3,5,6-fluoro benzyl halide for compounds 47 - 50 was increasingly difficult with increasing length of the oligomers (Scheme 5). Notably, for the synthesis of compounds $\mathbf{4 7}$ - 50, the reaction conditions needed to be carefully tuned (e.g. the base, temperature, the benzyl halide employed, the use of a microwave reactor).

\subsubsection{Ion transport}

The ion transport activity of compounds $\mathbf{4 7}-\mathbf{5 0}$ equipped with halogen bond donors, and the anion- $\boldsymbol{\pi}$ interactions-enabled control compounds $\mathbf{5 1}$ - $\mathbf{5 4}$ was evaluated using the HPTS assay under identical conditions as the ones used for the small molecules described in the last section. A clear increase in transport activity with increasing oligomeric length was evidenced, (Figure 7a,b) with a maximal activity measured for the octamer 54 with an $E C_{50}=110 \mathrm{nM}$ (i.e. a transporter/lipid molar ratio of $0.01 \%$ ). This transport activity is more than 2'300-times higher than the activity of monomeric iodo-perfluoro benzene $\mathbf{2 3}$.

Regarding the increase of transport activity observed from the monomer to the octamer, it would be normal to observe an increase in activity with increasing number of halogen bond donors. Nevertheless, the increase in transport activity was clearly not linear (see Table 3) as would be expected for a purely statistic effect. The authors found that the increase of transport activity could be described, as a function of the number of oligomeric repeats $(N)$, by the relationship described by Eq. 5:

$$
E C_{50} \propto N^{-m}
$$


Where $m$ is the cooperativity coefficient. For the halogen-bonding series $\mathbf{4 7}-\mathbf{5 0}$, a remarkably high value of cooperativity $m=3.37$ was obtained. Such a large cooperativity coefficient is well beyond the cooperativity typically observed for oligomers and polymers $(1<m<2)$ [73]. The Hill coefficient for all four oligomers $(\mathbf{4 7}-\mathbf{5 0})$ were measured to be around $n=1$, clearly indicating a different anion transport mechanism as compared to the monomer $(\mathbf{2 3} ; n=3.6)$. Together, the high transport activity, the unusually high cooperativity coefficient, the evidence of a fundamentally different anion transport mechanism, and the previous studies using $p$ olygophenyl rigid rods, strongly suggest that anion transport proceeds through an anion hoping mechanism involving halogen-bonding (Figure 7). The increased transport activity was also accompanied by a reduced anion selectivity, as would be expected due to the change in anion transport mechanism from a carrier to a channel. Nevertheless, a mild preference for the smaller halides was in line with a halogen-bonding based mechanism.

The success achieved by using the p-oligophenyl scaffold, with either hydrogen and halogen bonding, made it unsurprising when activity was also detected with the anion- $\pi$ versions of the oligomeric rigid rods $(\mathbf{5 1}-\mathbf{5 4})$. The observed transport activities were, however, more than an order of magnitude weaker than those measured for their halogen-bonding counterparts (see Table 3), and the calculated (using Eq. 5) cooperativity coefficient was less impressive ( $m=$ 2.13) and close to the expected value for multivalent systems. It is interesting, however, that the monomeric anion- $\pi$ control (perfluorobenzene 35 ) was completely inactive suggesting and confirming that to activate anion transport with this non-covalent interaction, either stronger $\pi$ acidic surfaces [21,39], or more complex molecular constructions [22,74] are required.

Table 3. Summary of transport activity for halogen bonding ion channels.[a]

\begin{tabular}{cccccc}
\hline \multicolumn{5}{c}{ Covalent approach: Rigid-rod $p$-oligophenyls } \\
\hline $\mathrm{XB}$ & $E C 50 / \mu \mathrm{M}[\mathrm{b}]$ & $n[\mathrm{c}]$ & Anion- $\pi$ & $E C 50 / \mu \mathrm{M}[\mathrm{b}]$ & $n[\mathrm{c}]$ \\
\hline $\mathbf{4 7}$ & $9.2 \pm 0.8$ & $1.0 \pm 0.2$ & $\mathbf{5 1}$ & $32.5 \pm 3.4$ & $1.1 \pm 0.1$ \\
$\mathbf{4 8}$ & $0.72 \pm 0.04$ & $1.0 \pm 0.1$ & $\mathbf{5 2}$ & $12.6 \pm 3.0$ & $0.8 \pm 0.1$ \\
$\mathbf{4 9}$ & $0.13 \pm 0.01$ & $1.3 \pm 0.1$ & $\mathbf{5 3}$ & $1.3 \pm 0.5$ & $0.5 \pm 0.1$ \\
$\mathbf{5 0}$ & $0.11 \pm 0.02$ & $0.8 \pm 0.1$ & $\mathbf{5 4}$ & $2.9 \pm 0.4$ & $0.6 \pm 0.1$ \\
\hline & Supramolecular approach: Pore forming peptides \\
\hline \multicolumn{5}{c}{$E C 50 / \mu \mathrm{M}[\mathrm{b}]$} & $n[\mathrm{c}]$ \\
\hline
\end{tabular}




\begin{tabular}{llllll}
\hline $\mathbf{6 0}$ & 2.37 & 3.5 & $\mathbf{6 3}$ & $>1000$ & n.d. \\
$\mathbf{6 1}$ & 0.39 & 3.6 & $\mathbf{6 4}$ & 0.32 & 3.3 \\
$\mathbf{6 2}$ & 0.93 & 3.1 & & & \\
\hline
\end{tabular}

[a] For the original data, see reference [70], [68], and [75]. [b] Effective concentration needed to reach 50\% activity in the HPTS assay (100 mM NaCl, $10 \mathrm{mM} \mathrm{HEPES,} \mathrm{pH}=7)$.

[c] Hill coefficient. n.d.: not determined.

\subsection{Halogen-bonding self-assembled pores and channels}

The molecular design presented in the previous section conclusively showed the suitability of halogen bonding in anion transport systems because good transport activities could be obtained for what is arguably the most efficient ion transport mechanism: an ion channel. Nevertheless, while the ensemble of studies carried out point towards a channel mechanism, this was not evidenced by conductance experiments in planar bilayer membranes. Moreover, the synthetic effort necessary to prepare octamer 50 (14 steps synthesis) makes it unlikely that such compound could be used beyond proof-of-concept experiments. Both these short comings were addressed with the introduction of a self-assembling artificial chloride channel using halogenbonding as one of the recognition interaction [68]. These channels were based on mono-peptide motifs developed earlier by the same group for their gelling properties [76], and can be readily prepared.

\subsubsection{Synthesis}

The development of these pore-forming monopeptides was based on the folding and association of $\alpha$ helices, which typically exploit hydrophobic lateral van der Waals interactions to form quaternary structures in proteins. The authors hypothesized that having a peptidic backbone with lateral hydrophobic interactions could lead to self-assembled fibers, an approach that was proven correct to prepare gels. Accordingly, the development of the channel/pore forming compounds that will be described in the following was based on a combinatorial approach: a common backbone was proposed (see Scheme 6) and the combination of five amino acids (A, F, V, I, and L) and alkyl chains were prepared.

The synthesis of the Library required first the preparation of the iodo-perfluorophenyl pendant chain. Here, 2,3,5,6-tetrafluoro-4-iodophenol 55 was coupled to methyl 2-bromoacetate. Subsequent deprotection of methylester 56 using $\mathrm{NaOH}$ and $\mathrm{HCl}$ provided free-acid 57. This compound could be coupled with the corresponding peptidic backbone $\mathbf{5 9}$ using benzotriazol- 
1-yloxytris(dimethylamino)phosphonium hexafluorophosphate (BOP) giving access to all 15 compounds of the Library in moderate to high yields in gram scale.

\subsubsection{Ion transport}

The full Library was tested in the HPTS assay - which further demonstrates the convenience of this technique for screening ion transport activity - and the best compounds $(60-62$, Scheme 7) were selected for further studies. From this screening, alanine, leucine, and phenylalanine gave the best results with a clear preference for leucine compounds. It is important to note that the length of the alkyl chain $\left(\mathrm{R}_{1}\right)$ can completely change the activity of the compounds from very active to almost inactive by seemingly minor changes (e.g. $\mathrm{R}_{1}=\mathrm{C}_{10} \mathrm{H}_{21}$ or $\mathrm{C}_{12} \mathrm{H}_{25}$ ).

To be able to compare the activity of the newly reported channels with the results reported by Matile and co-workers [51,70], the authors not only used the same conditions for the key measurements in the HPTS assay, but in addition they re-evaluated the transport activity of iodo-perfluorohexane $\mathbf{2 8}$ finding quasi-identical results to the ones previously reported [51] thus confirming the remarkable suitability of halogen bonding in anion transport. Under these conditions, they found ion transport activities as high $E C_{50}=0.39 \mu \mathrm{M}$ (for 61, see Table 3) which are remarkable for such simple compounds.

A number of control experiments were conducted to gain insights into the transport mechanism of these supramolecular ion transporters: The SPQ assay (see Help Box 1) permitted to assess directly chloride transport; the HPTS assay in presence of a small concentration of FCCP (a proton channel) confirmed that anions were preferentially transported over protons; the HPTS assay in presence of a small concentrations of valinomycin (a potassium carrier) were presented as evidence against a $\mathrm{H}_{+} / \mathrm{M}_{+}$antiport mechanism. Overall, LUVs experiment suggested that transport proceeds through an $\mathrm{A}_{-} / \mathrm{OH}_{-}$antiport mechanism with a minor $\mathrm{M}_{+} / \mathrm{A}_{-}$symport component.

Ion selectivity was also explored by the sequential exchange of the external cations and anions. Exchange of the cations in the external buffer in the HPTS assay had no measurable effect in the measured activity. The anion exchange of the salt in the external buffer led to intriguing results: While a clear selectivity for chloride was measured for compound $\mathbf{6 0}$, compound $\mathbf{6 1}$ was selective for iodine and compound $\mathbf{6 2}$ - which was only poorly selective - showed a preference for bromine. This suggests that not only binding but also size matters for transport, and that each compound is strongly influenced by their molecular structure. 
To further confirm the importance of halogen bonding for the activity of the putative channels, compound 63 (which lacks both $\pi$-acidity and halogen bond donors) was also tested under the same condition but only poor to negligible ion transport activity was measured. Nevertheless, in a subsequent paper published the same year [75], the authors reported on the formation of chloride pores for a family of monopeptides similar to the halogen-bonded collection presented here. From those studies, compound $\mathbf{6 4}$ was the most active with an ion transport activity of $E C_{50}=270 \mathrm{nM}$. They concluded that ion transport, in that particular, case was due to the formation of pores, possibly formed by the assembly of $3 \times 8$ monomers 64 , which did not require the halogen-bonding to achieve a better transport activity [75].

\subsubsection{Conductance experiments in planar lipid bilayers}

Based on the crystal structures available for similar compounds, the authors hypothesized that compounds 60 - 62 could form self-assembled oligomers that could function as ion channels. The evidence obtained in the HPTS assay did support this hypothesis and the authors carried out conductance experiment in planar lipid bilayer membranes using compound 61. A very distinct step-like behavior was observed (Figure 5b) and a Cl- conductance of $\gamma_{\mathrm{Cl}^{-}}=586 \mathrm{fS}$ was found from variable voltage experiments. The authors indicated that the open probability of the channel is low due to the dynamic nature of the channel.

\subsubsection{Molecular dynamics simulations}

Similarly, based on the crystal structures of a similar compound [76], the authors hypothesized that the active structure could assemble in a similar fashion in the membrane and in the solid state. From this assumption, they constructed a molecular model using the hydrogen-bonded peptide structure. This non-optimized array of eight 61 units was then inserted into a $70 \times 70 \times 74$ Å box containing 128 1-palmitoyl-2-oleoyl-sn-glycero-3-phosphocoline (POPC) and $\mathrm{KCl}(0.15$ M) to simulate the membrane. Using the CHARMM program, the evolution of the system was simulated over $>200 \mathrm{ps}$ at $303.15 \mathrm{~K}$ and the last $20 \mathrm{~ns}$ (1000 structures) were analyzed. The results, that can be summarized by Figure 8a, show a) that the structure remains linked by hydrogen bonding; b) the halogen-bond donors do not remain aligned but are still in close proximity, and c) the misalignment of the halogen-bond units suggests that with only seven, or even six units, the supramolecular structure might have enough reach to span the bilayer membrane (DOPC ca. $2.71 \mathrm{~nm}[77])$.

\subsubsection{Anticancer activity}


The remarkably high transport activity of these channels/pores, motivated the authors to explore potential applications in cancer therapy, notably chemotherapy. To investigate this possibility, they incubated human breast cancer cells (BT-474 from the American type culture collection, USA) with compounds $\mathbf{6 0}$ and $\mathbf{6 1}$ using concentrations ranging from 0 to $100 \mu \mathrm{M}$. Both compounds reduced the cell viability significantly $(\sim 100 \%$ and $40 \%$ cell mortality for compounds 60 and 61 respectively at $100 \mu \mathrm{M}$ ) demonstrating that this family of compounds are active in vitro. It is worth mentioning that the anticancer activity was not directly corelated to the ion transport activity (where compound $\mathbf{6 1}$ was seven-times more active than compound 60), which might arise from differences in membrane composition.

\section{Discussion and Perspectives}

The addition of halogen bonding to the collection of non-covalent interactions that can be employed to achieve ion transport across lipid bilayer membranes can definitely be considered as accomplished. Although it is been less than ten years since halogen bonding was proposed and used towards enabling ion transport, significant and conclusive evidence has already been presented and further highlights how suitable this inconspicuous non-covalent interaction is for the task. Its strength and directionality, playing in coherence with its lipophilicity are presumably some of the reasons for its success. This is probably best shown by the use of small molecules (Section 3) to achieve anion transport. Indeed, in that particular case, hardly any other covalent or non-covalent interaction is involved and it represents, therefore, undisputable proof of the afore mentioned relevance and suitability of halogen bonding. Such examples are necessary when demonstrating a conceptual work and exist also for other interactions (e.g. phase transfer using the tetrabutylammonium cation, crown ethers, and so forth). As such, there is a clearly defined line separating actual applications and the conceptual work.

However, to include halogen bonding in active functional materials for ion transport is necessarily a more complex situation. The examples that have been reported, which are all summarized here, are without exception significantly harder to interpret. They all result from a wider combination of interactions, having structural and functional purposes. In most examples, it was possible to prove that the presence of halogen-bond donors had a distinguishable effect on the transport activity reported, notably with regards to anion selectivity, but required additional assistance for complementary interactions. This was notably the case for the macrocyclic systems which required either ion pairing or hydrogen bonding to show any transport activity. However, such a situation is all too common for natural and artificial functional materials alike: Functional activity is the result of an intricate interplay of forces. 
This delicate equilibrium can and has been exploited in many ways. Although, it was not explicitly discussed in this chapter, halogen bonding has an important effect on membrane permeation [78], which can also enable the transport of actual (macro)molecules [79,80]. To these additional functional properties, it is important to add the arrival of other sigma-hole interactions [26] which have been proven as good and relevant as halogen bonding. Chalcogen [27,28] and Pnictrogen [29] bonding have thus also enriched the library of non-covalent interactions that can promote ion transport. While the landscape of possibilities has certainly become wider, the future of halogen-bonding - and sigma-hole interactions in general - is more promising than ever.

\section{Summary}

This chapter has discussed comprehensively the use of halogen bonding in transmembrane ion transport systems. Over the past ten years, examples of anion carriers and channels owing their functional activity to this non-covalent interaction have been reported. The active involvement of halogen bonding in transmembrane anion transport can be easily understood when considering the nature of this arguably less common interaction: Its high directionality, strength, and a rather hydrophobic nature are favorable for this application.

Three main categories have been defined, based on their structural features, but that are directly related by their mode of action. First, macrocyclic approaches have been described. Here, calix[4]arenes and oxacalix[2]arene[2]triazine frameworks have been used. In both cases, a combination of halogen bonding with other non-covalent interactions was necessary to activate ion transport. This was the result of an arguably sub-optimal molecular design: while these transporters were based on macrocyclic arrays, the arrangement of the pendant halogen-bond donors did not allow for a perfectly concentric coordination of the anion as shown by DFT calculations (for calix[4]arenes) and crystallography (for oxacalix[2] arene[2]triazine). In spite of that, good transport activities, up to $E C 50=32 \mu \mathrm{M}$ for calix 11, could be measured when using a mild halogen-bond donor (ortho-iodophenyl). Moreover, the oxacalix[2]arene[2] triazine analogues were shown to display anticancer activity demonstrating the robustness of the functional principle.

The best evidence for the functional relevance of halogen bonding in anion transport came from comparatively simpler molecules. A series of perfluorinated iodo arenes and alkanes were shown to self-assemble in the lipid bilayer membrane permitting the translocation of anions. The driving force was proposed to be the formation of multiple halogen bonds to the anion. This concept was most beautifully demonstrated by the weak, yet detectable, transport activity 
$\left(E C_{50}=1.5 \mathrm{mM}\right)$ of trifluoroiodomethane 32 which with only five atoms and a boiling point of $-22{ }^{\circ} \mathrm{C}$ has little else to offer other that a strong halogen-bonding nature. Good transport activities were measured for perfluoro-1-iodohexane $\mathbf{2 8}\left(E_{50}=3.1 \mu \mathrm{M}\right)$, with high

permeability ratios $\left(\frac{\mathrm{P}_{\mathrm{Cl}^{-}}}{\mathrm{P}_{\mathrm{K}^{+}}}\right)$further confirming a clean anion transport mechanism.

To further improve the ion transport activity, two different strategies towards ion channels were reported. On a first approach, a synthetically demanding $p$-octaphenyl rigid rod bearing eight halogen-bond donors (50) was prepared and nanomolar activities could be achieved $\left(E C_{50}=\right.$ $110 \mathrm{nM}$ ). The strongly cooperative nature of the halogen-bond donors was advanced as evidence for a putative ion channel mechanism. On a complementary approach, pore-forming monopeptides were functionalized with halogen-bond donors. In this case, the self-assembly in the lipid bilayer membrane by hydrogen bonding and hydrophobic interactions of these, easily accessible, monopeptides (e.g. 61) led to the formation of an ordered array of halogen-bond donors with high anion transport activities $(E C 50=390 \mathrm{nM})$. Here, conductance experiments in planar lipid bilayer membranes further confirmed the formation of channels/pores.

Together, these examples, albeit limited in number, span the range of passive ion transport mechanisms and conclusively demonstrate the relevance of halogen bonding in transmembrane ion transport. The recent involvement of new research groups to the development of these systems is promising towards achieving active transport which would represent a major breakthrough in the field. Moreover, the development of explicitly mixed systems involving traditional non-covalent interactions and other sigma-hole interactions will certainly strengthen the development of ion transport in general. Overall, the examples we described here nicely highlight the relevance of halogen bonding in solution and aim to motivate new discoveries.

\section{Acknowledgments}

We would like to express our gratitude to our co-workers and collaborators. In particular, the contributions the groups of J. Mareda (Geneva) and P. Metrangolo and G. Resnati (Milan) are warmly acknowledged. The CNRS and the University of Geneva are acknowledged for financial support.

\section{References}

[1] Skou, J. C., Biochim. Biophys. Acta 1957, 23, 394-401.

[2] Tabushi, I., Kuroda, Y., Yokota, K., Tetrahedron Lett. 1982, 23, 4601-4604.

[3] Sakai, N., Matile, S., Langmuir 2013, 29, 9031-9040.

[4] Sisson, A. L., Shah, M. R., Bhosale, S., Matile, S., Chem. Soc. Rev. 2006, 35, 12691286.

[5] Matile, S., Som, A., Sordé, N., Tetrahedron 2004, 60, 6405-6435. 
[6] Matile, S., Vargas Jentzsch, A., Montenegro, J., Fin, A., Chem. Soc. Rev. 2011, 40, 2453-2474.

[7] Chui, J. K. W., Fyles, T. M., Chem. Soc. Rev. 2012, 41, 148-175.

[8] Gale, P. A., Davis, J. T., Quesada, R., Chem. Soc. Rev. 2017, 46, 2497-2519.

[9] Gale, P. A., Acc. Chem. Res. 2011, 44, 216-226.

[10] Busschaert, N., Gale, P. A., Angew. Chem. Int. Ed. 2013, 52, 1374-1382.

[11] Gokel, G. W., Daschbach, M. M., Coord. Chem. Rev. 2008, 252, 886-902.

[12] Tanaka, Y., Kobuke, Y., Sokabe, M., Angew. Chem. Int. Ed. 1995, 34, 693-694.

[13] Chui, J. K. W., Fyles, T. M., Org. Biomol. Chem. 2014, 12, 3622-3634.

[14] Sakai, N., Brennan, K. C., Weiss, L. A., Matile, S., J. Am. Chem. Soc. 1997, 119, 8726-8727.

[15] Tedesco, M. M., Ghebremariam, B., Sakai, N., Matile, S., Angew. Chem. Int. Ed. 1999, 38, 540-543.

[16] Fyles, T. M., Chem. Soc. Rev. 2007, 36, 335-347.

[17] Díaz de Greñu, B., Hernández, P. I., Espona, M., Quiñonero, D., Light, M. E., Torroba, T., Pérez-Tomás, R., Quesada, R., Chem. Eur. J. 2011, 17, 14074-14083.

[18] Saggiomo, V., Otto, S., Marques, I., Felix, V., Torroba, T., Quesada, R., Chem. Commun. 2012, 48, 5274-5276.

[19] Li, X., Shen, B., Yao, X.-Q., Yang, D., J. Am. Chem. Soc. 2009, 131, 13676-13680.

[20] Seganish, J. L., Santacroce, P. V., Salimian, K. J., Fettinger, J. C., Zavalij, P., Davis, J. T., Angew. Chem. Int. Ed. 2006, 45, 3334-3338.

[21] Dawson, R. E., Hennig, A., Weimann, D. P., Emery, D., Ravikumar, V., Montenegro, J., Takeuchi, T., Gabutti, S., Mayor, M., Mareda, J., Schalley, C. A., Matile, S., Nat. Chem. 2010, 2, 533-538.

[22] Adriaenssens, L., Estarellas, C., Vargas Jentzsch, A., Martinez Belmonte, M., Matile, S., Ballester, P., J. Am. Chem. Soc. 2013, 135, 8324-8330.

[23] Gorteau, V., Bollot, G., Mareda, J., Perez-Velasco, A., Matile, S., J. Am. Chem. Soc. 2006, 128, 14788-14789.

[24] Vargas Jentzsch, A., Hennig, A., Mareda, J., Matile, S., Acc. Chem. Res. 2013, 46, 2791-2800.

[25] Sakai, N., Sordé, N., Das, G., Perrottet, P., Gerard, D., Matile, S., Org. Biomol. Chem. 2003, 1, 1226-1231.

[26] Lim, J. Y. C., Beer, P. D., Chem 2018, 4, 731-783.

[27] Benz, S., Macchione, M., Verolet, Q., Mareda, J., Sakai, N., Matile, S., J. Am. Chem. Soc. 2016, 138, 9093-9096.

[28] Macchione, M., Tsemperouli, M., Goujon, A., Mallia, A. R., Sakai, N., Sugihara, K., Matile, S., Helv. Chim. Acta 2018, 101, e1800014.

[29] Lee, L. M., Tsemperouli, M., Poblador-Bahamonde, A. I., Benz, S., Sakai, N., Sugihara, K., Matile, S., J. Am. Chem. Soc. 2019, 141, 810-814.

[30] Lieffrig, J., Jeannin, O., Frąckowiak, A., Olejniczak, I., Świetlik, R., Dahaoui, S., Aubert, E., Espinosa, E., Auban-Senzier, P., Fourmigué, M., Chem. Eur. J. 2013, 19, 14804-14813.

[31] Zhu, Z., Wang, G., Xu, Z., Chen, Z., Wang, J., Shi, J., Zhu, W., Phys. Chem. Chem. Phys. 2019, 21, 15106-15119.

[32] Hennig, A., Fischer, L., Guichard, G., Matile, S., J. Am. Chem. Soc. 2009, 131, 16889-16895.

[33] Sarwar, M. G., Dragisic, B., Sagoo, S., Taylor, M. S., Angew. Chem. Int. Ed. 2010, 49, 1674-1677.

[34] Beale, T. M., Chudzinski, M. G., Sarwar, M. G., Taylor, M. S., Chem. Soc. Rev. 2013, 42, 1667-1680. 
[35] Chudzinski, M. G., McClary, C. A., Taylor, M. S., J. Am. Chem. Soc. 2011, 133, 10559-10567.

[36] Caballero, A., White, N. G., Beer, P. D., Angew. Chem. Int. Ed. 2011, 50, 1845-1848.

[37] Caballero, A., Zapata, F., White, N. G., Costa, P. J., Felix, V., Beer, P. D., Angew. Chem. Int. Ed. 2012, 51, 1876-1880.

[38] Kilah, N. L., Wise, M. D., Serpell, C. J., Thompson, A. L., White, N. G., Christensen, K. E., Beer, P. D., J. Am. Chem. Soc. 2010, 132, 11893-11895.

[39] Vargas Jentzsch, A., Emery, D., Mareda, J., Metrangolo, P., Resnati, G., Matile, S., Angew. Chem. Int. Ed. 2011, 50, 11675-11678.

[40] Huang, W.-L., Wang, X.-D., Li, S., Zhang, R., Ao, Y.-F., Tang, J., Wang, Q.-Q., Wang, D.-X., J. Org. Chem. 2019, 84, 8859-8869.

[41] Sidorov, V., Kotch, F. W., Kuebler, J. L., Lam, Y.-F., Davis, J. T., J. Am. Chem. Soc. 2003, 125, 2840-2841.

[42] Iqbal, K. S. J., Cragg, P. J., Dalton Trans. 2007, 85, 26-32.

[43] Abraham, W., J. Incl. Phenom. Macrocycl. Chem. 2002, 43, 159-174.

[44] Rathore, R., Abdelwahed, S. H., Guzei, I. A., J. Am. Chem. Soc. 2004, 126, 1358213583.

[45] Matile, S., Sakai, N., in Analytical Methods in Supramolecular Chemistry, Vol. 1 (ed. Schalley, C.A.), Wiley-VCH, Weinheim, Germany 2012, pp. 711-742.

[46] Behr, J. P., Kirch, M., Lehn, J.-M., J. Am. Chem. Soc. 1985, 107, 241-246.

[47] Li, S., Wang, X.-D., Wang, Q.-Q., Ao, Y.-F., Wang, D.-X., Wang, M.-X., Crystal Growth \& Design 2016, 16, 5460-5465.

[48] Wang, X.-D., Li, S., Ao, Y.-F., Wang, Q.-Q., Huang, Z.-T., Wang, D.-X., Org. Biomol. Chem. 2016, 14, 330-334.

[49] Luo, J., Ao, Y.-F., Wang, Q.-Q., Wang, D.-X., Angew. Chem. Int. Ed. 2018, 57, $15827-15831$.

[50] Xu, R.-B., Wang, Q.-Q., Ao, Y.-F., Li, Z.-Y., Huang, Z.-T., Wang, D.-X., Org. Lett. 2017, 19, 738-741.

[51] Vargas Jentzsch, A., Emery, D., Mareda, J., Nayak, S. K., Metrangolo, P., Resnati, G., Sakai, N., Matile, S., Nat. Commun. 2012, 3, 905.

[52] Pike, M. M., Simon, S. R., Balschi, J. A., Springer, C. S., Proc. Natl. Acad. Sci. USA 1982, 79, 810-814.

[53] Koulov, A. V., Lambert, T. N., Shukla, R., Jain, M., Boon, J. M., Smith, B. D., Li, H., Sheppard, D. N., Joos, J.-B., Clare, J. P., Davis, A. P., Angew. Chem. Int. Ed. 2003, 42, 4931-4933.

[54] Davis, A. P., Sheppard, D. N., Smith, B. D., Chem. Soc. Rev. 2007, 36, 348-357.

[55] Litvinchuk, S., Sordé, N., Matile, S., J. Am. Chem. Soc. 2005, 127, 9316-9317.

[56] Stadler, E., Dedek, P., Yamashita, K., Regen, S. L., J. Am. Chem. Soc. 1994, 116, 6677-6682.

[57] Lolkema, J. S., Slotboom, D.-J., J. Gen. Physiol. 2015, 145, 565-574.

[58] Wissing, F., Smith, J. A. C., J. Membrane Biol. 2000, 177, 199-208.

[59] Verkman, A. S., Takla, R., Sefton, B., Basbaum, C., Widdicombe, J. H., Biochemistry 1989, 28, 4240-4244.

[60] Nostro, Lo, P., Ninham, B. W., Chem. Rev. 2012, 112, 2286-2322.

[61] Spence, G. T., Beer, P. D., Acc. Chem. Res. 2013, 46, 571-586.

[62] Mangel, M., Jendrasiak, G. L., Chem. Phys. Lipids 1976, 16, 167-180.

[63] Klotz, K. H., Benz, R., Biophys. J. 1993, 65, 2661-2672.

[64] Mueller, P., Rudin, D. O., Tien, H. T., Wescott, W. C., Nature 1962, 194, 979-980.

[65] Montal, M., Mueller, P., Proc. Natl. Acad. Sci. USA 1972, 69, 3561-3566.

[66] Pulcu, G. S., Galenkamp, N. S., Qing, Y., Gasparini, G., Mikhailova, E., Matile, S., Bayley, H., J. Am. Chem. Soc. 2019, 141, 12444-12447. 
[67] Goto, C., Yamamura, M., Satake, A., Kobuke, Y., J. Am. Chem. Soc. 2001, 123, 12152-12159.

[68] Ren, C., Ding, X., Roy, A., Shen, J., Zhou, S., Chen, F., Yau Li, S. F., Ren, H., Yang, Y. Y., Zeng, H., Chem. Sci. 2018, 9, 4044-4051.

[69] Sakai, N., Houdebert, D., Matile, S., Chem. Eur. J. 2003, 9, 223-232.

[70] Vargas Jentzsch, A., Matile, S., J. Am. Chem. Soc. 2013, 135, 5302-5303.

[71] Weiss, L. A., Sakai, N., Ghebremariam, B., Ni, C., Matile, S., J. Am. Chem. Soc. 1997, 119, 12142-12149.

[72] Sakai, N., Majumdar, N., Matile, S., J. Am. Chem. Soc. 1999, 121, 4294-4295.

[73] Hennig, A., Gabriel, G. J., Tew, G. N., Matile, S., J. Am. Chem. Soc. 2008, 130, 10338-10344.

[74] Perez-Velasco, A., Gorteau, V., Matile, S., Angew. Chem. Int. Ed. 2008, 47, 921-923.

[75] Ren, C., Zeng, F., Shen, J., Chen, F., Roy, A., Zhou, S., Ren, H., Zeng, H., J. Am. Chem. Soc. 2018, 140, 8817-8826.

[76] Ren, C., Shen, J., Chen, F., Zeng, H., Angew. Chem. Int. Ed. 2017, 56, 3847-3851.

[77] Guo, Y., Pogodin, S., Baulin, V. A., J. Chem. Phys. 2014, 140, 174903.

[78] Govindaraj, V., Ungati, H., Jakka, S. R., Bose, S., Mugesh, G., Chem. Eur. J. 2019, $25,11180-11192$.

[79] Gerebtzoff, G., Li-Blatter, X., Fischer, H., Frentzel, A., Seelig, A., ChemBioChem 2004, 5, 676-684.

[80] Wang, T., Yin, P., Yang, Y., Yin, W., Zhang, S., Yang, M., Qin, Y., Ma, Y., Lei, Z., Ma, H., ACS Sustain. Chem. Eng. 2019, 7, 6295-6303.

Figures:
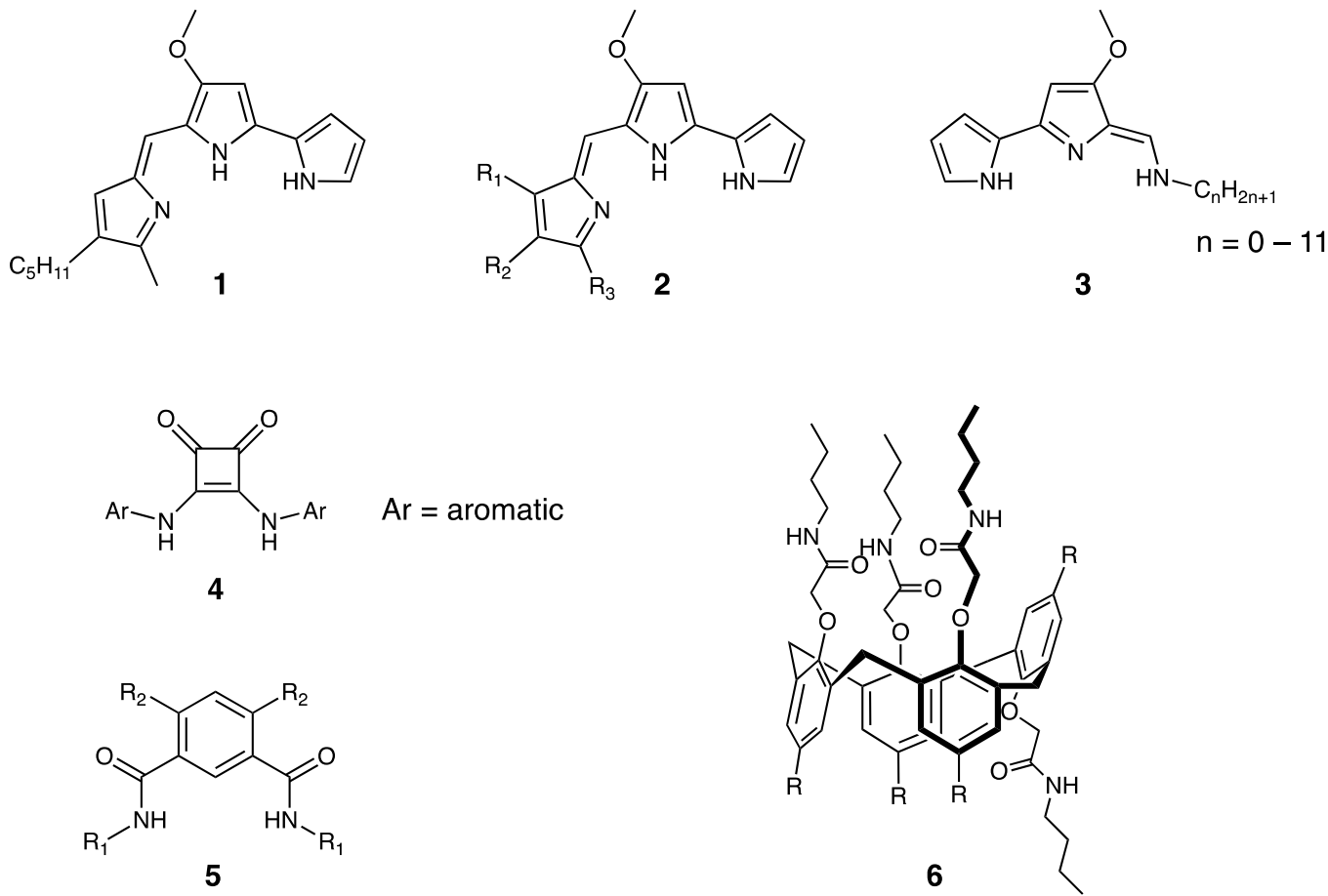

Scheme 1. Examples of artificial and naturally occurring anion transporters. 

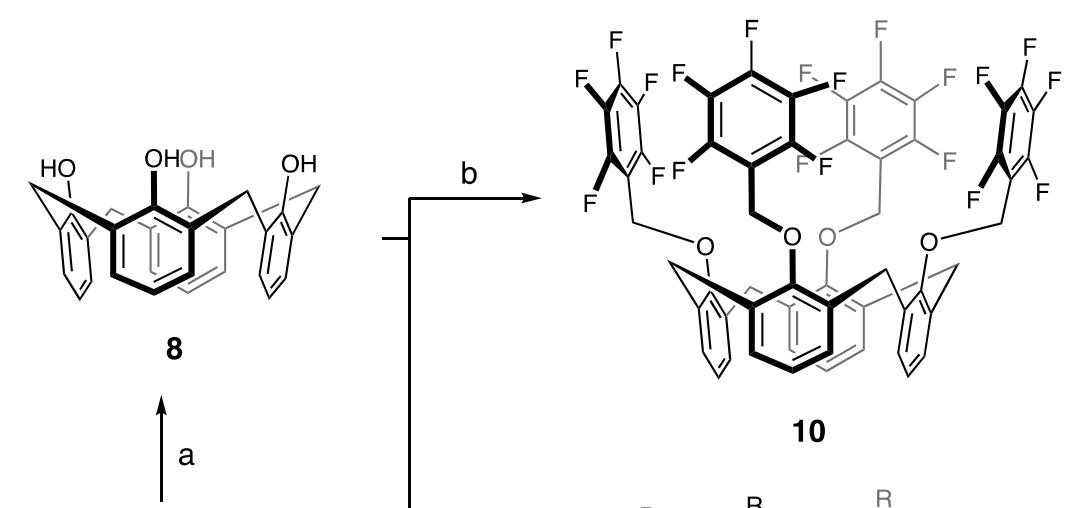

10
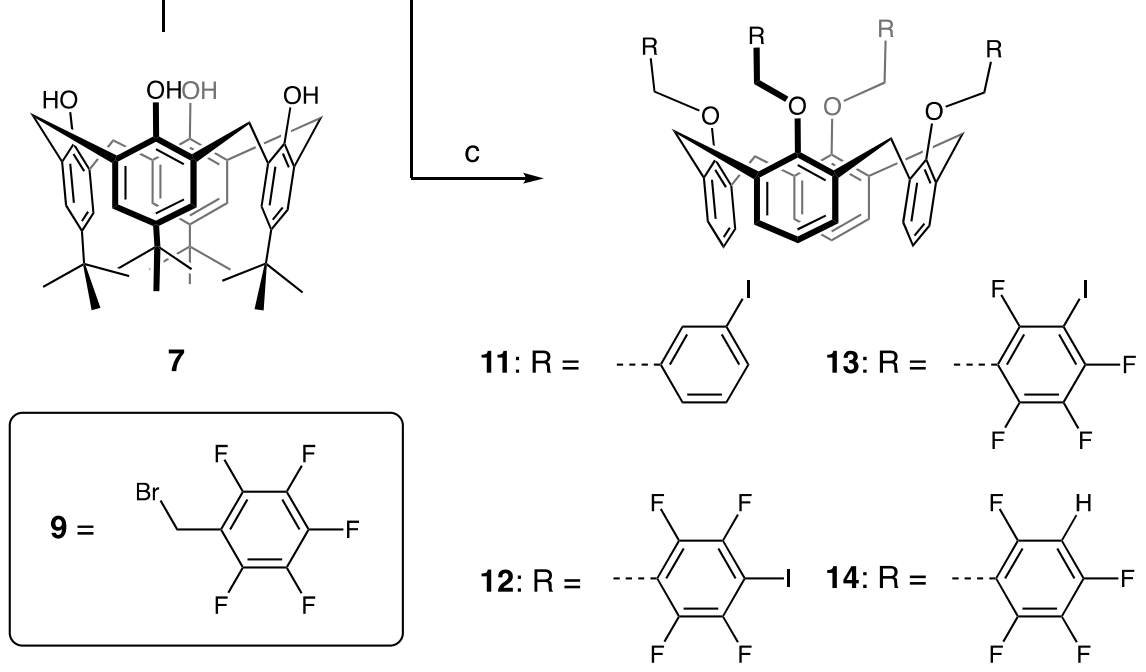

Scheme 2. Synthesis of calix[4]arene based transporters $\mathbf{1 0}$ - 13. a) $\mathrm{AlCl}_{3}$, phenol, toluene, rt, $1.5 \mathrm{~h}, 70 \%$; b) 9, $\mathrm{NaH}, \mathrm{DMF},-10{ }^{\circ} \mathrm{C}, 5 \mathrm{~min}, 86 \%$; c) Corresponding benzyl halide, $\mathrm{NaH}, \mathrm{DMF}$, $-10-80{ }^{\circ} \mathrm{C}, 15-120 \min (\mathbf{1 1}, 73 \% ; \mathbf{1 2}, 45 \% ; \mathbf{1 3}, 65 \% ; \mathbf{1 4}, 70 \%)[39,44]$. 
a

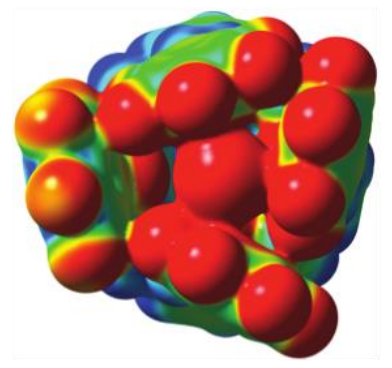

d

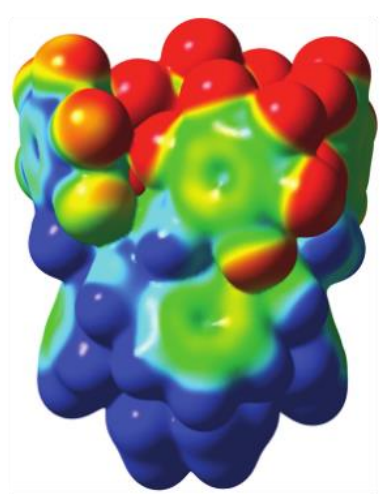

g

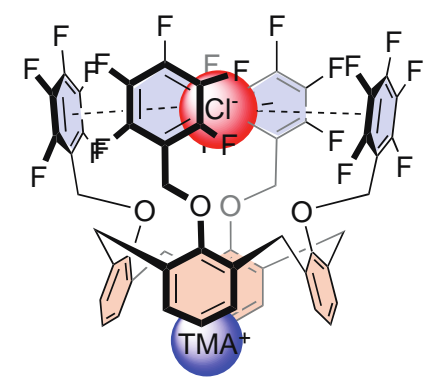

10-TMACI b

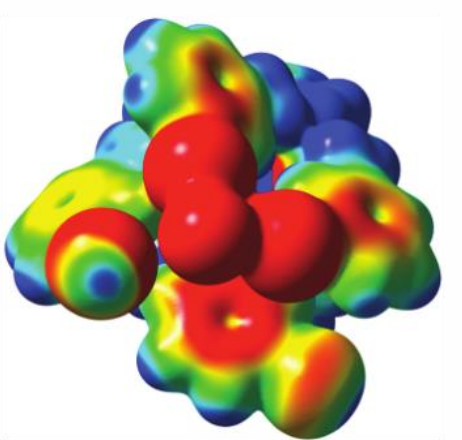

e

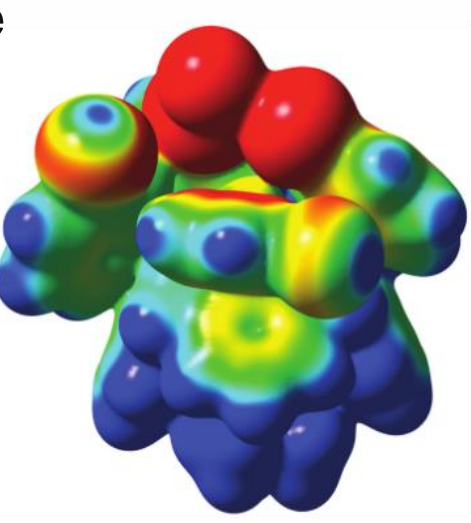

h
C

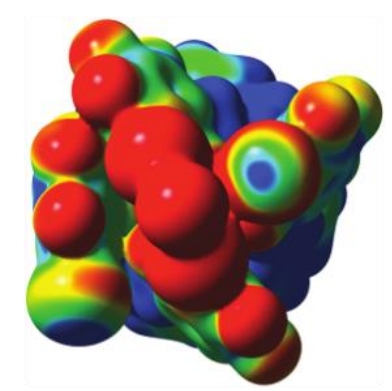

f

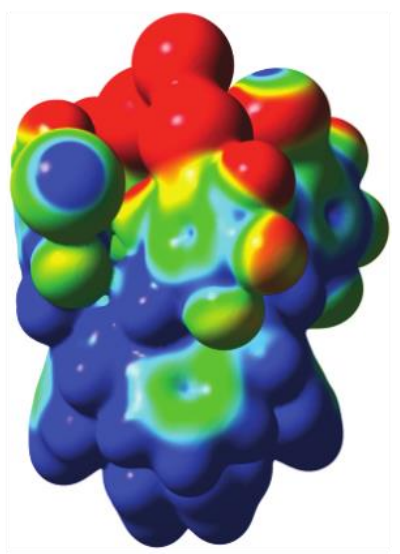

i

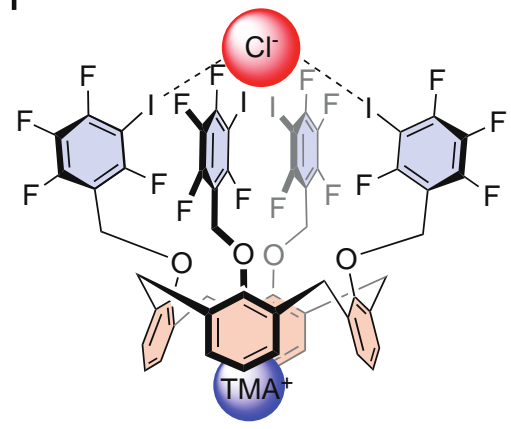

13.TMACI

Figure 1. a-f) DTF (PBE1PBE/6-311G**) optimized TMACl complexes of calix[4]arenes 10 $(\mathrm{a}, \mathrm{d}), \mathbf{1 1}(\mathrm{b}, \mathrm{e})$, and $\mathbf{1 3}$ (c,f) with electrostatic potential surfaces (blue positive, red negative, \pm 25 kcal mol-1; isodensity: 0.05 a.u.); g-i) Schematic representations of the complexes. Adapted with permission from [39]. Copyright 2011 Wiley. 
<smiles>[X]c1cccc([X])c1O</smiles>

15<smiles>[X]c1cccc([X])c1Oc1nc(Cl)nc(Cl)n1</smiles><smiles>CC(=O)NC1CCCCC1(C)NC(C)=O</smiles>

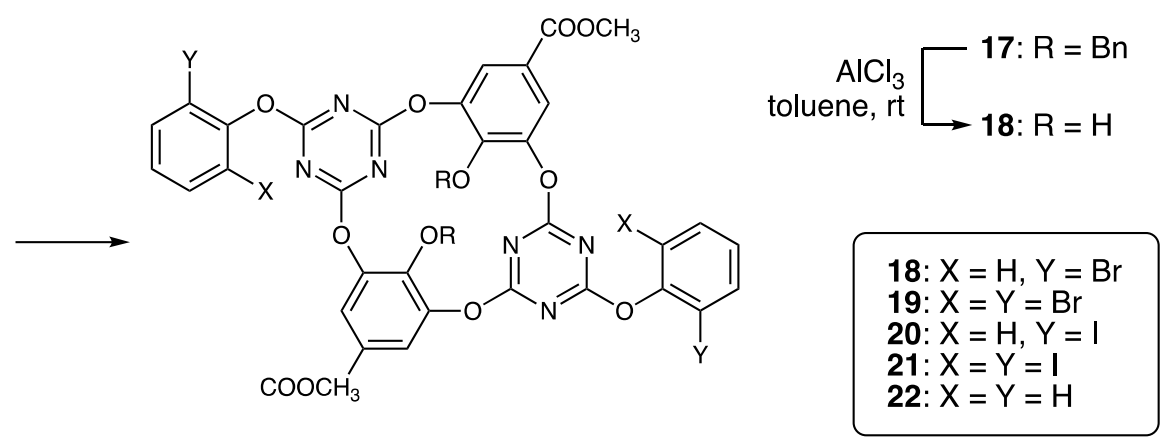

Scheme 3. Synthesis of oxacalix[2]-arene[2]triazine based transporters $\mathbf{1 8}-\mathbf{2 2}$. The control macrocycle 22 was prepared following a different reaction path [40].

a
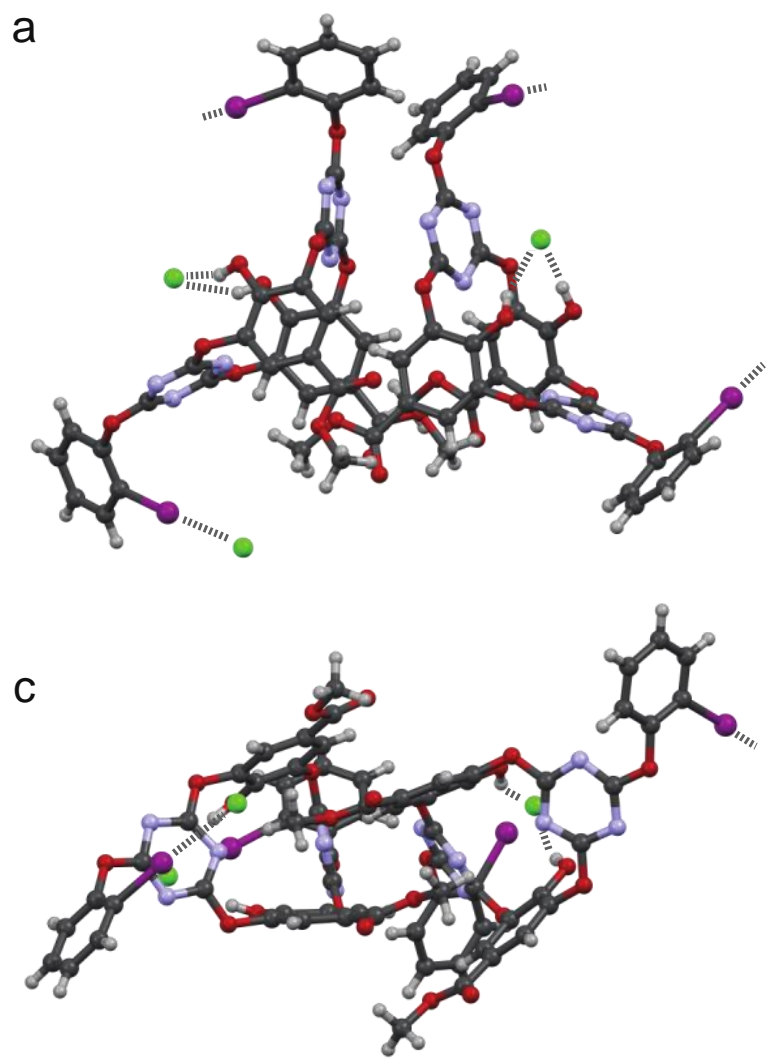

b

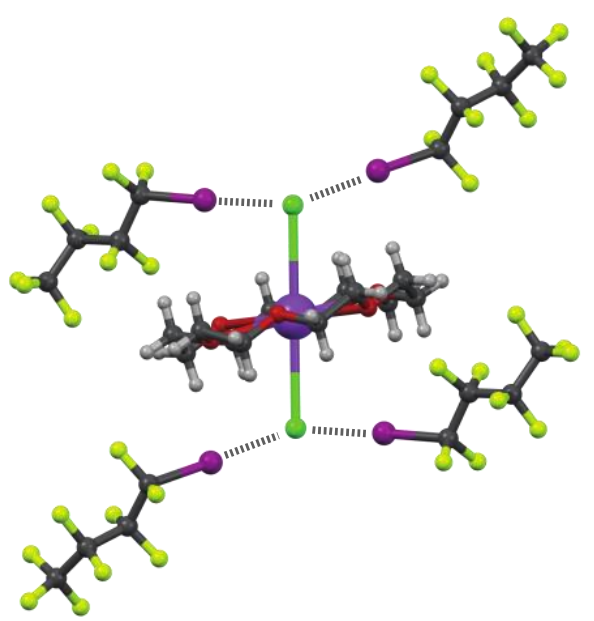

d

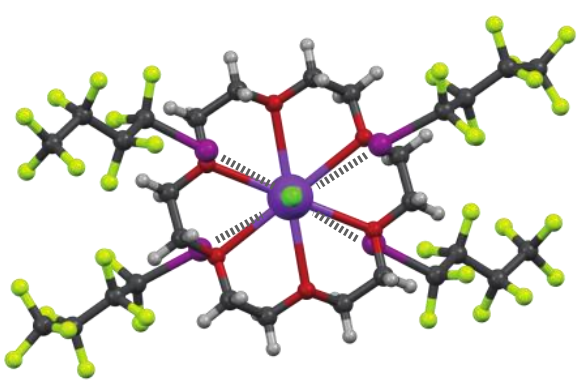

Figure 2. Examples of the use of crystallography to help elucidate the role of halogen bonding in anion transport. a,c) Side and top view of oxacalix[2]-arene[2]triazine 20 co-crystalized with TBACl. Adapted with permission from [40]. Copyright 2019 American Chemical Society. b,d) 
Side and top view of iodo-perfluorobutane 29 co-crystalized with $\mathrm{KCl}$ and 18-crown-6. Adapted with permission from [51]. Copyright 2012 Nature Publishing Group.

a

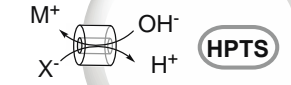

HPTS:

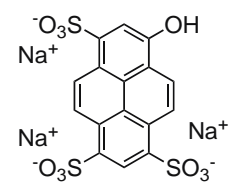

b

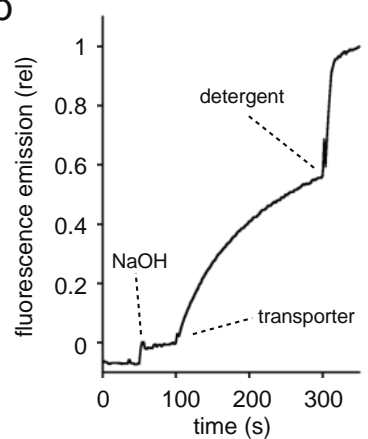

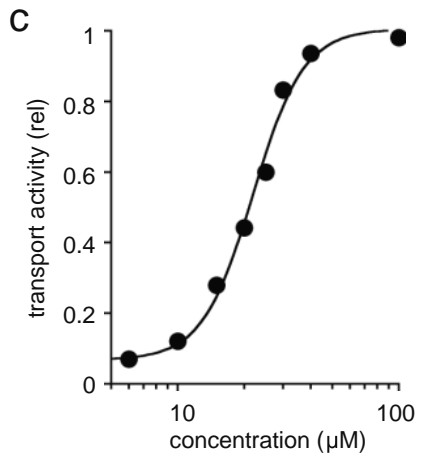

Figure 3. The HPTS assay. a) Schematic representation of the HPTS assay in LUVs.

b) A typical fluorescent trace obtained for a single HPTS experiment. c) A typical dose-response curve that allows to determine the EC50 value for a given system.

a
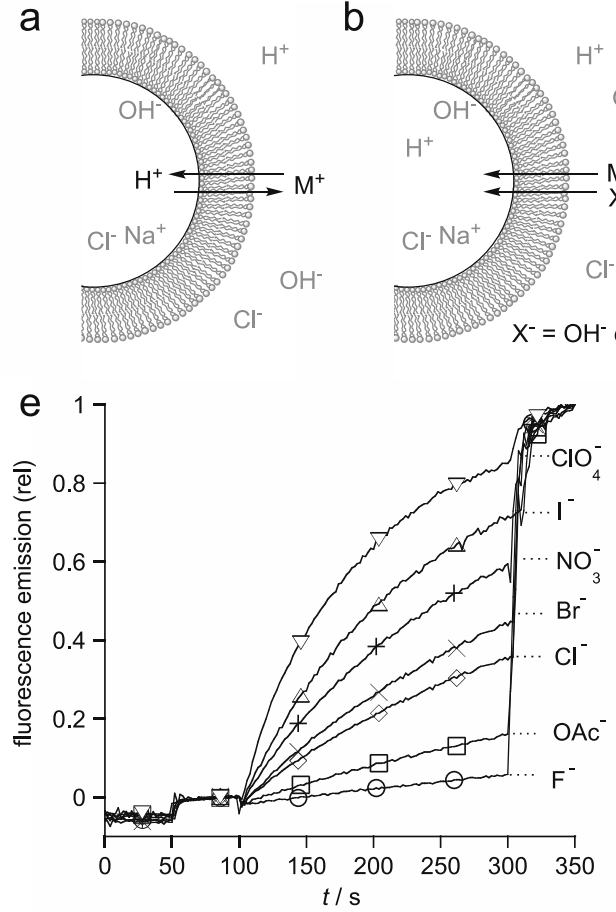

C
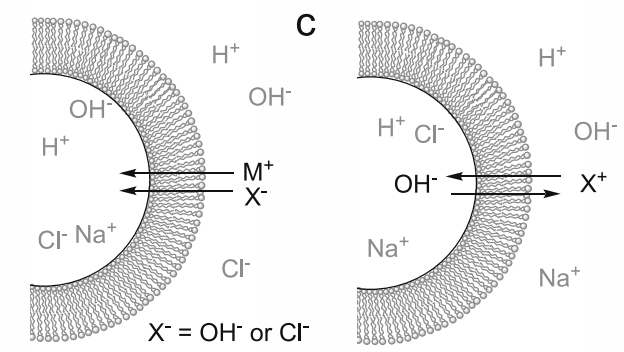

f

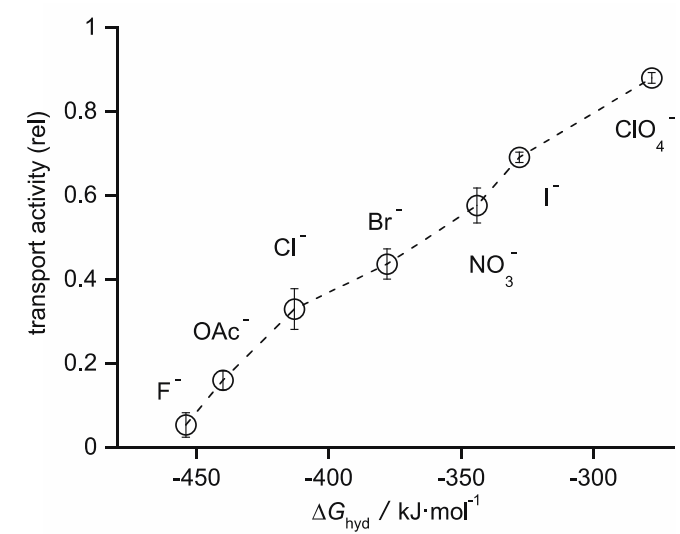


Figure 4. Schematic representation of symport and antiport transport mechanisms and the determination of ion selectivity. a) Cation-proton antiport; b) Cation-anion symport; c) Anion-hydroxy antiport; d) Anion-cation symport; e) An example of an idealized fluorescent trace obtained during selectivity studies using the HPTS assay; f) An anion selectivity topology displaying a Hofmeister selectivity. Disclaimer: e and $f$ are simulated data.

a. lodo-perfluoro and bromo-perfluoro arenes<smiles>Fc1c(F)c(F)c(I)c(F)c1F</smiles>

23<smiles>Fc1c(F)c(I)c(I)c(F)c1I</smiles>

24<smiles>Fc1c(F)c(I)c(F)c(F)c1I</smiles>

25<smiles>Fc1c(F)c(F)c(Br)c(F)c1F</smiles>

26

b. lodo-perfluoro alkanes

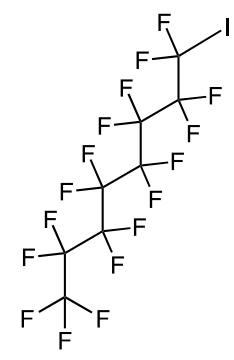

27

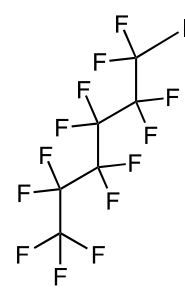

28

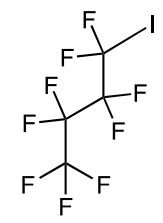

29

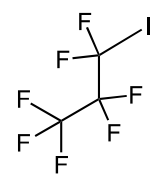

30<smiles>FC(F)(F)C(I)(I)C(F)(F)F</smiles>

31<smiles>FC(F)(F)I</smiles>

32

c. control systems

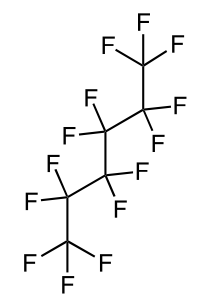

33

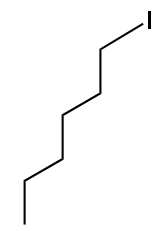

34<smiles>Fc1c(F)c(F)c(F)c(F)c1F</smiles>

35<smiles>C[n+]1cc(I)ccc1I</smiles>

36<smiles>Ic1ccccc1</smiles>

37

Scheme 4. Molecular structures of the small molecules tested as halogen-bonding based anion transporters. 
a

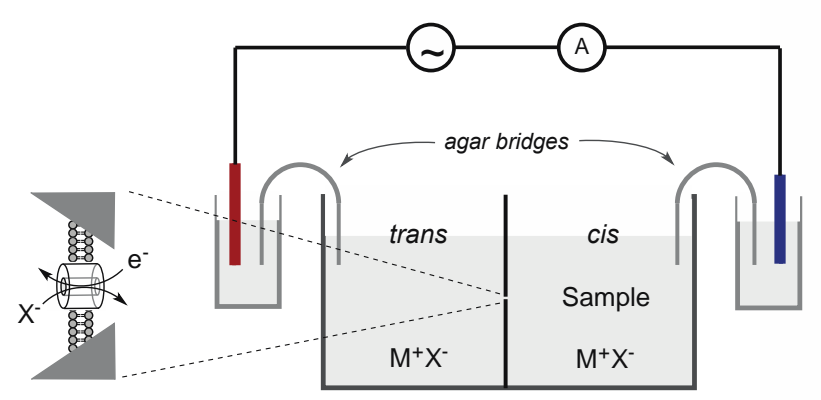

C

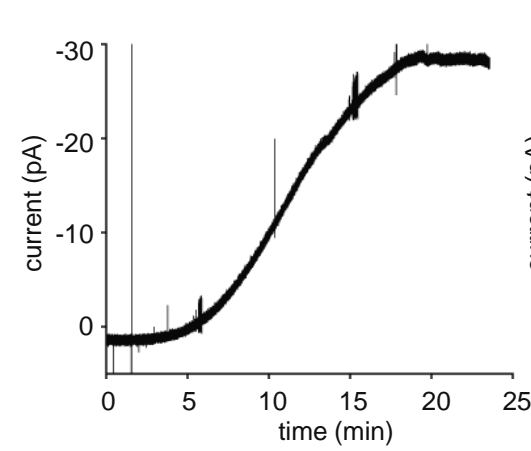

d b

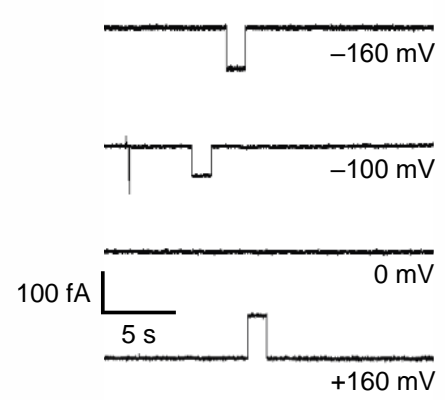

e

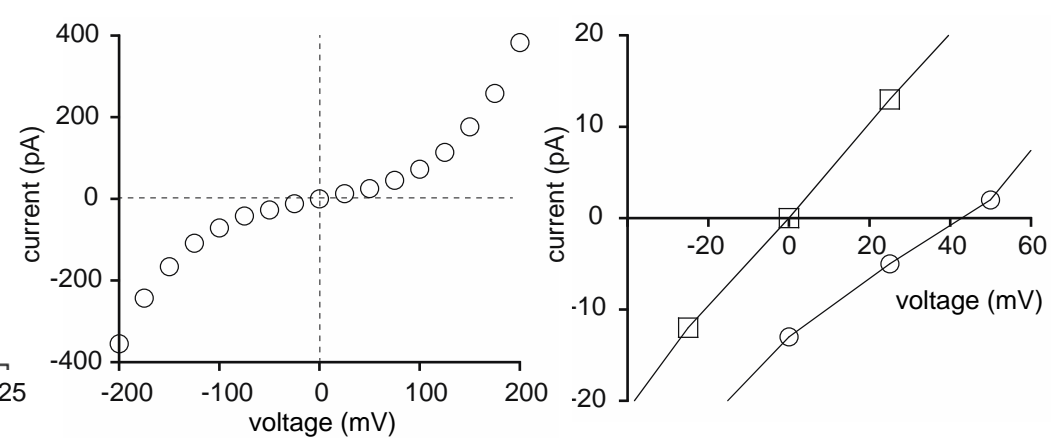

Figure 5. Conductance experiments in planar lipid bilayer membranes. a) Typical experimental set-up used to measure conductance in planar bilayer experiments; b) Single current trace of $\mathbf{6 1}$ $(3.6 \mu \mathrm{M})$ recorded at an applied voltage of $-160,-100,0$, and $+160 \mathrm{mV}$ with symmetric chambers (cis and trans chambers $1 \mathrm{M} \mathrm{KCl}$ ). Adapted with permission from [68]. Copyright 2018 The Royal Society of Chemistry; c) Current $I$ measured in conductance experiments in response to the addition of iodo-perfluorobenzene $\mathbf{2 3}$ to the cis chamber under a constant applied voltage of $-50 \mathrm{mV}(2 \mathrm{M} \mathrm{KCl}$ cis and trans $)$. d) Current $I$ as a function of the applied voltage ( -200 to $+200 \mathrm{mV} ; 500 \mu \mathrm{M} 23$ cis; $2 \mathrm{M} \mathrm{KCl}$ in both cis and trans); e) $I-V$ profile in presence of $23(500 \mu \mathrm{M}$ cis) under symmetric ( $2 \mathrm{M} \mathrm{KCl}$ cis and trans; circles) or asymmetric ( $2 \mathrm{M} \mathrm{KCl}$ cis and $0.25 \mathrm{M} \mathrm{KCl}$ in trans; squares) conditions to determine the permeability ratio. Adapted with permission from [51]. Copyright 2012 Nature Publishing Group. 

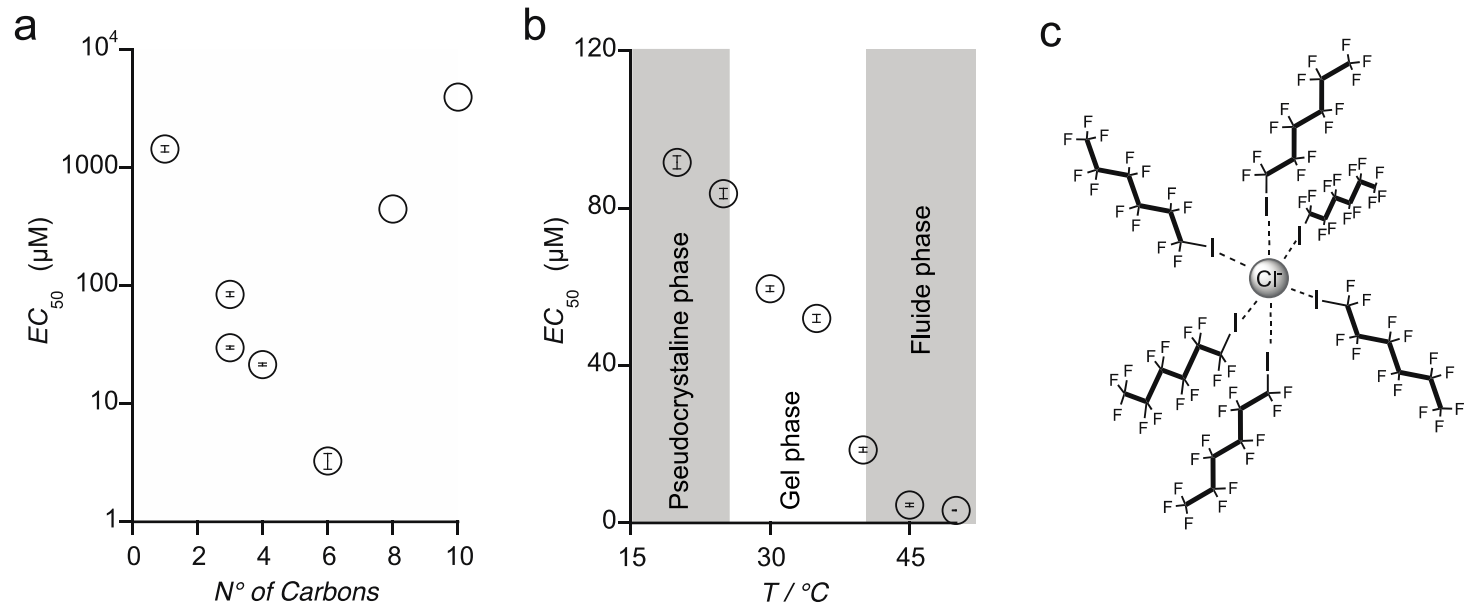

Figure 6. a) Evolution of the effective molar concentration to achieve 50\% transport activity $(E C 50)$ in the HPTS assay with increasing chain length for the iodo-perfluoro alkane series (i.e. 32, 31, 30, 29, 28, 27, and 26 respectively); b) Evolution of the EC50 values for iodo perfluorobutane $\mathbf{2 9}$ as a function of temperature in the HPTS assay using DPPC lipids; c) Schematic representation of the idealized supramolecular structure that was proposed as active species. Adapted with permission from [51]. Copyright 2019 Nature Publishing Group. 
<smiles>COc1cc(-c2ccc([N+](=O)Cl)c(OC)c2)ccc1[N+](=O)Cl</smiles>

38

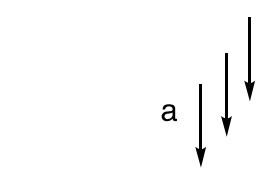<smiles>Cc1ccc(-c2ccc(C)cc2OCC(=O)O)c(OCC(=O)O)c1</smiles>

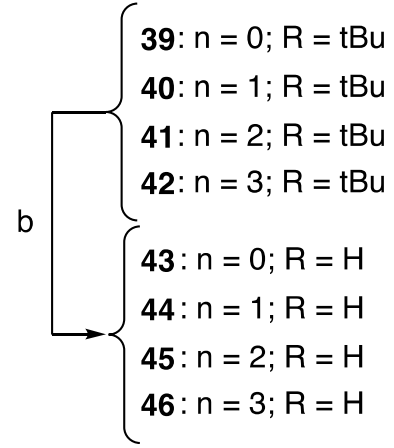<smiles>C=C(C)c1cccc(OCC(=O)OCc2c(F)c(F)c(I)c(F)c2F)c1</smiles><smiles>C=C(C)c1ccc(-c2ccccc2OCC(=O)OCc2c(C)cc(C)c(F)c2F)c(OCC(=O)OCc2c(F)c(F)c(I)c(F)c2F)c1</smiles><smiles>O=C(COc1ccccc1)OCc1c(F)cc(I)c(F)c1F</smiles>

47: $\mathrm{n}=0$

48: $\mathrm{n}=1$

49: $n=2$

$50: n=3$<smiles>C=C(COc1cccc(C(C)C)c1)OCC(=O)OCC(=O)OCc1c(F)c(F)c(F)c(F)c1COc1ccc(-c2ccc(C(C)c3cccc(OCC(=O)OCc4c(F)c(F)c(F)c(F)c4F)c3)cc2OCC(=O)OCc2c(F)c(F)c(F)c(F)c2F)c(F)c1F</smiles>

Scheme 5. Synthesis of rigid-rods $p$-oligophenyls based transporters 47 - 54. a) The synthesis of 2, 4, 6 and $8 p$-oligophenyls was achieved following the previously described procedure [14] in 4 to 10 steps; b) TFA, DCM, 1-3 h, quant.; c) Corresponding benzyl halide, DIPEA and/or $\mathrm{Na}_{2} \mathrm{SO}_{4}$ (anh), DMF, $(\mu \mathrm{W})$, between $120{ }^{\circ} \mathrm{C}$ and $170{ }^{\circ} \mathrm{C}, 15 \mathrm{~min}$ to $24 \mathrm{~h}$, yields between $30 \%$ and 80\%.; d) 9, DIPEA and/or $\mathrm{Na}_{2} \mathrm{SO}_{4(\text { anh), }} \mathrm{DMF},(\mu \mathrm{W})$, between $-10{ }^{\circ} \mathrm{C}$ and $120{ }^{\circ} \mathrm{C}, 1 \mathrm{~h}$ to $3 \mathrm{~h}$, yields between 50\% and quant [70]. 
a

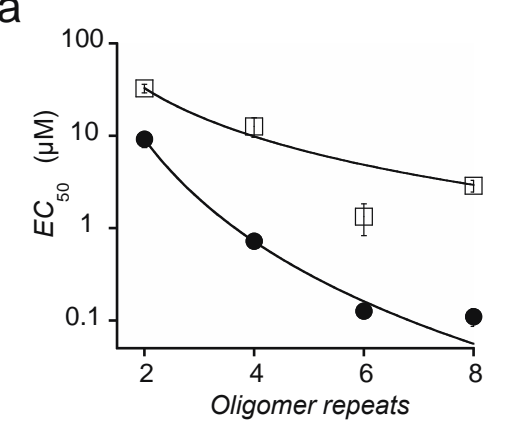

b

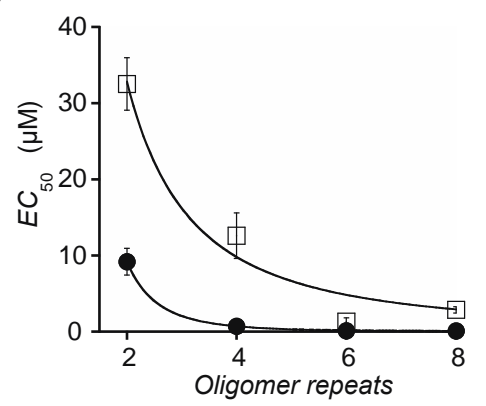

C

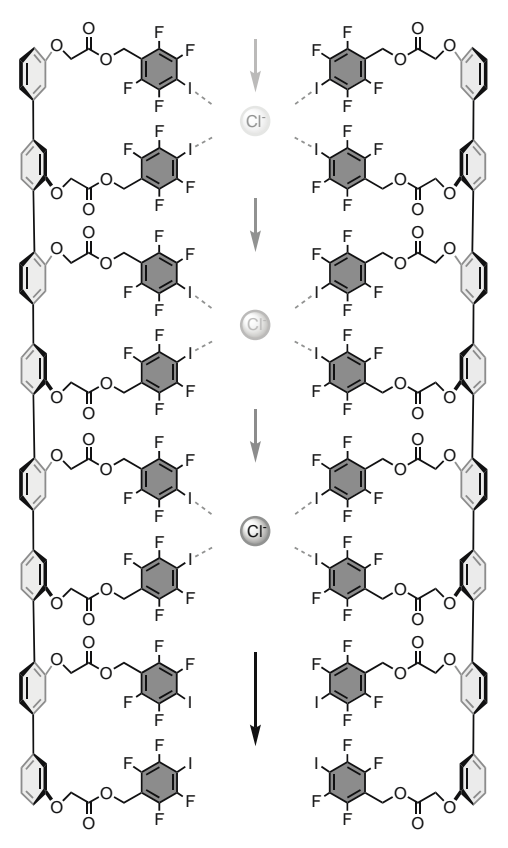

Membrane

Figure 7. a,b) Evolution of the effective molar concentration to achieve 50\% transport activity $(E C 50)$ in the HPTS assay with increasing oligomer size; logarithmic (a) and linear (b) y-axis; c) Schematic representation of the proposed active structure with rigid rod $\mathbf{5 0}$ showing anion hopping across the membranes. Adapted with permission from [70]. Copyright 2013 American Chemical Society.<smiles>Oc1c(F)c(F)c(I)c(F)c1F</smiles>

55<smiles>COC(=O)CBr</smiles><smiles>COC(=O)COc1c(F)c(F)c(I)c(F)c1F</smiles>

56 $\begin{aligned} & \text { 1. } \mathrm{NaOH}, \mathrm{MeOH}, \\ & \text { reflux, } 1 \mathrm{~h}\end{aligned}$
$\stackrel{\text { 2. } \mathrm{HCl}(1 \mathrm{M})}{\longrightarrow}$

2. $\mathrm{HCl}(1 \mathrm{M})$<smiles>O=C(O)COc1c(F)c(F)c(I)c(F)c1F</smiles>

57<smiles>[R7]NC(=O)C([R7])NC(=O)OCC1c2ccccc2-c2ccccc21</smiles><smiles>[R7]NC(=O)[C@H]([R7])NC(=O)COc1c(F)c(F)c(F)c(F)c1F</smiles>

$$
\begin{aligned}
& \mathrm{R}_{1}=n-\mathrm{C}_{8} \mathrm{H}_{17} \quad \mathrm{R}_{2}=\mathrm{CH}_{3} \text { (Ala, } \mathbf{A} \text { ) } \\
& n-\mathrm{C}_{10} \mathrm{H}_{21} \quad \mathrm{CH}_{2} \mathrm{C}_{6} \mathrm{H}_{5} \text { (Phe, F) } \\
& n-\mathrm{C}_{12} \mathrm{H}_{25} \quad \mathrm{CH}\left(\mathrm{CH}_{3}\right)_{2} \text { (Val, V) } \\
& \mathrm{CH}\left(\mathrm{CH}_{3}\right) \mathrm{C}_{2} \mathrm{H}_{5} \text { (Ile, I) } \\
& \mathrm{CH}_{2} \mathrm{CH}\left(\mathrm{CH}_{3}\right)_{2} \text { (Leu, L) }
\end{aligned}
$$


Scheme 6. Synthesis of the Library of pore-forming peptides tested by Zeng and co-workers [68].

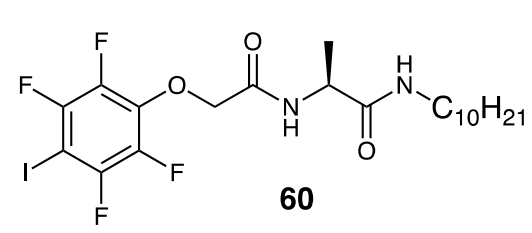<smiles>CC(C)CC(NC(=O)COc1c(F)c(F)c(I)c(F)c1F)C(=O)Nc1ccccc1</smiles><smiles>CCCCCCCCNC(=O)[C@H](CC(C)C)NC(=O)COc1c(F)c(F)c(F)c(F)c1F</smiles>

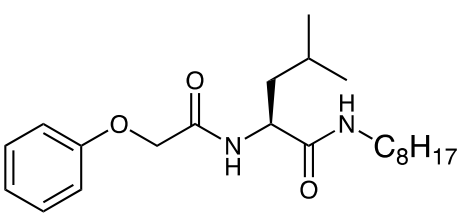

63

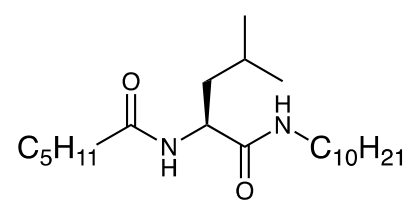

64

Scheme 7. Molecular structures of the pore-forming peptides used by Zeng and co-workers $[68,75]$.

a

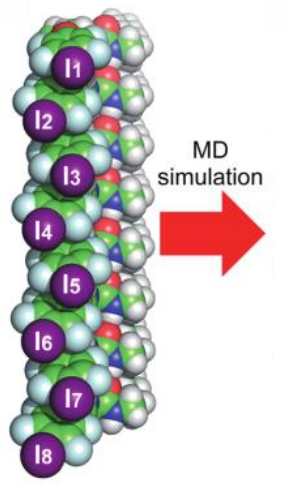

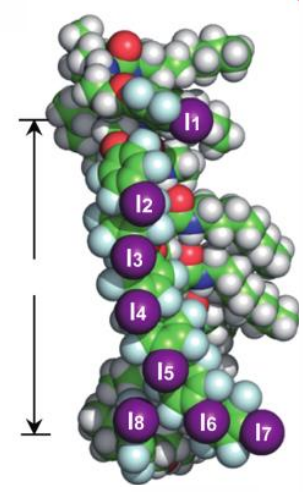

b

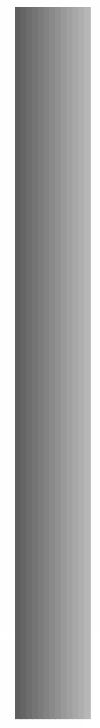

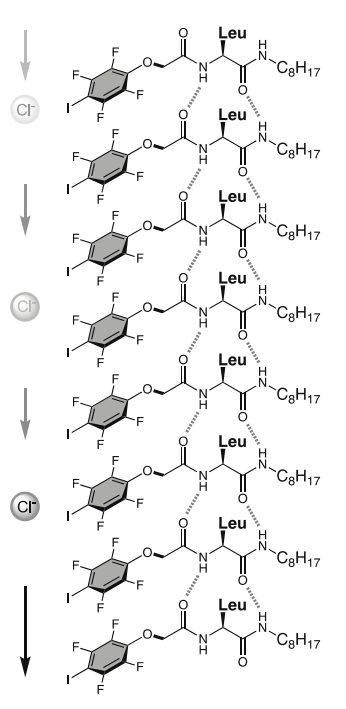

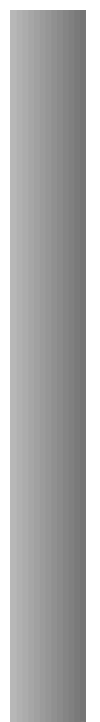

Figure 8. a) Computationally optimized H-bonded octamer formed from pore-forming peptide 61; the structures prior and after MD simulations are shown. Adapted with permission from [68]. Copyright 2018 The Royal Society of Chemistry; b) Schematic representation of the proposed active structure with self-assembled peptides 61. 